\title{
List-coloring claw-free graphs with $\Delta-1$ colors
}

\author{
Daniel W. Cranston* Landon Rabern ${ }^{\dagger}$
}

May 2, 2018

\begin{abstract}
We prove that if $G$ is a quasi-line graph with $\Delta(G)>\omega(G)$ and $\Delta(G) \geq 69$, then $\chi_{O L}(G) \leq \Delta(G)-1$. Together with our previous work, this implies that if $G$ is a claw-free graph with $\Delta(G)>\omega(G)$ and $\Delta(G) \geq 69$, then $\chi_{\ell}(G) \leq \Delta(G)-1$.
\end{abstract}

\section{Introduction}

Brooks' Theorem shows that to color a graph $G$ with $\Delta(G)$ colors, the obvious necessary condition (no clique of size $\Delta(G)+1$ ) is also sufficient, when $\Delta(G) \geq 3$. Borodin and Kostochka [5] conjectured something similar for $\Delta(G)-1$ colors.

Conjecture 1.1 (Borodin-Kostochka [5]). If $G$ is a graph with $\Delta(G) \geq 9$ and no clique of size $\Delta(G)$, then $\chi(G) \leq \Delta(G)-1$.

This conjecture is a major open problem and has been the subject of much research. Reed [21] used probabilistic techniques to prove the conjecture when $\Delta(G) \geq 10^{14}$. For graphs with smaller maximum degree, the best result [11] is that $\chi(G) \leq \Delta(G)-1$ whenever $G$ has no clique of size $\Delta(G)-3$. We have also proved Conjecture 1.1 for claw-free graphs [10].

Although the Borodin-Kostochka conjecture is far from resolved, it is natural to pose the analogous conjectures for list-coloring and online list-coloring, replacing $\chi(G)$ in Conjecture 1.1 with $\chi_{\ell}(G)$ and $\chi_{O L}(G)$. These conjectures first appeared in print in [10] and [12], respectively. In the case of Brooks' Theorem, the analogues for $\chi_{\ell}(G)$ and $\chi_{O L}(G)$ both hold. In fact, the proof of the latter [16] constructs an orientation of $E(G)$ from which the result follows by the Alon-Tarsi Theorem. The present paper applies this approach to the Borodin-Kostochka conjecture. More precisely, given a graph with $\Delta(G) \geq 9$ and $\omega(G)<\Delta(G)$, we seek an orientation of $E(G)$ that implies that $\chi_{O L}(G) \leq \Delta(G)-1$. Our main result is the following.

Theorem 1.2. Let $G$ be a quasi-line graph with $\Delta(G) \geq 69$. If $\omega(G)<\Delta(G)$, then $\chi_{O L}(G) \leq \Delta(G)-1$. Further, Painter has a natural winning strategy, using a combination of the Alon-Tarsi Theorem and the kernel method.

\footnotetext{
*Department of Mathematics and Applied Mathematics, Viriginia Commonwealth University, Richmond, VA; dcranston@vcu.edu; Research of the first author is partially supported by NSA Grant 98230-15-1-0013.

${ }^{\dagger}$ LBD Data Solutions, Lancaster, PA; landon.rabern@gmail.com
} 
Chudnovsky and Seymour [8, 9] proved a structure theorem for claw-free graphs. Although it is rather complicated, it implies the following structure theorem for quasi-line graphs, which is much simpler. (We define the undefined terms in Section 1.1.)

Theorem 1.3 ([8, 9]). Every connected quasi-line graph not containing a non-linear homogeneous pair of cliques is a circular interval graph or a composition of linear interval strips.

Theorem 1.3 suggests a natural approach to prove Theorem 1.2. Let $G$ be a quasi-line graph with $\Delta(G) \geq 69$ and $\omega(G)<\Delta(G)$. In Sections 2.1 and 2.2 we show that if $G$ is a minimal counterexample to our theorem, then $G$ cannot be a circular interval graph and $G$ cannot contain a non-linear homogeneous pair of cliques. In Section [2.3, we consider compositions of linear interval strips (which include line graphs, as a special case). We show that a minimal counterexample $G$ must be formed from a line graph $G^{\prime}$ by deleting some (possibly empty) matching $M$. Further, we can choose $G^{\prime}$ such that $\Delta\left(G^{\prime}\right)=\Delta(G)$ and $\omega\left(G^{\prime}\right)<\Delta(G)$. So we prove the desired result for all quasi-line graphs if we prove it for line graphs. Finally, in Section 3 we prove the theorem for line graphs. By combining this result with Theorem 5.6 from [10], we get that every claw-free graph $G$ with $\Delta(G) \geq 69$ and $\omega(G)<\Delta(G)$ satisfies $\chi_{\ell}(G) \leq \Delta(G)-1$. In other words, for these graphs we prove the list-coloring version of the Borodin-Kostochka conjecture.

It is somewhat surprising that line graphs seem to be the most difficult case in the proof. In our reduction from general quasi-line graphs to line graphs, we only need $\Delta(G) \geq 9$. It is our proof of Theorem 1.2 for line graphs that requires $\Delta(G) \geq 69$. As noted above, Theorem 5.6 in [10] shows that if $\chi_{\ell}(G) \leq \Delta(G)-1$ for all quasi-line graphs $G$ with $\omega(G)<$ $\Delta(G)$ and $\Delta(G) \geq 9$, then the same bound holds for all such claw-free graphs. In unpublished work, we have extended this reduction to online list-coloring. Thus, $\chi_{O L}(G) \leq \Delta(G)-1$ for every such claw-free graph $G$ with $\Delta(G) \geq 69$. (And again, the hypothesis $\Delta(G) \geq 69$ is needed only for the case of line graphs.)

\subsection{Definitions}

Most of our terminology and notation are standard. We write $N(v)$ for the neighborhood of a vertex $v$, and $N[v]=N(v) \cup\{v\}$. When $u$ and $v$ are adjacent, we write $u \leftrightarrow v$; otherwise, $u \leftrightarrow v$. We write $[t]$ for $\{1, \ldots, t\}$ (but we reserve, for example, [13] for citations). The degree, $d(v)$, is the size of $N(v)$ and $d_{H}(v)$ is the size of $N(v) \cap V(H)$, for any subgraph $H$. A graph is $k$-degenerate if every subgraph $H$ contains a vertex $v$ with $d_{H}(v) \leq k$. The complement of $G$ is denoted $\bar{G}$. The maximum degree and clique number of $G$ are denoted $\Delta(G)$ and $\omega(G)$, and we may write $\Delta$ and $\omega$ when the context is clear. The chromatic number of $G$ is $\chi(G)$. Similarly, the list chromatic and online list-chromatic numbers are $\chi_{\ell}(G)$ and $\chi_{O L}(G)$. The edge chromatic number of $G$ is $\chi^{\prime}(G)$, and $\chi_{\ell}^{\prime}(G)$ and $\chi_{O L}^{\prime}(G)$ are defined analogously. A graph $G$ is $L$-colorable if $G$ has a proper coloring $\varphi$ such that $\varphi(v) \in L(v)$ for all $v \in V(G)$. A graph $G$ is $f$-choosable if $G$ is $L$-colorable whenever $|L(v)| \geq f(v)$ for all $v$, and $f$-paintable is defined analogously. We write $d_{1}$ for the function $f(v)=d(v)-1$, and thus define $d_{1}$-choosable and $d_{1}$-paintable.

The subgraph of a graph $G$ induced by vertex set $S$ is $G[S]$. The average degree, ad $(G)$, of a graph $G$ is $2|E(G)| /|V(G)|$. The maximum average degree, $\operatorname{mad}(G)$, is the $\operatorname{maximum}$ 
of $\operatorname{ad}(H)$, taken over all subgraphs $H$ of $G$. A graph or subgraph is complete if it induces a clique; otherwise it is incomplete. A graph is almost complete if deleting some vertex yields a complete graph. The join of graphs $G$ and $H$, denoted $G \vee H$, is formed from their disjoint union by adding every edge with one endpoint in each of $G$ and $H$.

A linear interval graph is one in which the vertices can be placed on the real line so that for each vertex $v$ its neighborhood is precisely the vertices in some interval of the line containing $v$. A circular interval graph is defined analogously, except that now the vertices are placed on the unit circle. The line graph $G$ of some graph $H$ has $V(G)=E(H)$ and $u v \in E(G)$ whenever $u, v \in V(G)$ and they correspond to edges in $H$ sharing an endpoint. A graph $G$ is quasi-line if for each vertex $v \in V(G)$, the subgraph $G[N(v)]$ can be covered by two cliques. A graph if claw-free if it contains no induced copy of $K_{1,3}$. It is easy to check that the class of claw-free graphs properly contains the class of quasi-line graphs, which in turn properly contains the class of line graphs.

A homogeneous pair of cliques $\left(A_{1}, A_{2}\right)$ in a graph $G$ is a pair of disjoint nonempty cliques such that for each $i \in\{1,2\}$, every vertex in $G \backslash\left(A_{1} \cup A_{2}\right)$ is either adjacent to all of $A_{i}$ or non-adjacent to all of $A_{i}$ and $\left|A_{1}\right|+\left|A_{2}\right| \geq 3$. The pair $\left(A_{1}, A_{2}\right)$ is non-linear if $G\left[A_{1} \cup A_{2}\right]$ contains an induced 4-cycle.

Chudnovsky and Seymour [8] generalized the class of line graphs by introducing the notion of compositions of strips ([18, Chapter 5] gives a more detailed introduction). We use the modified definition from King and Reed [19]. A strip $\left(H, A_{1}, A_{2}\right)$ is a claw-free graph $H$ containing two cliques $A_{1}$ and $A_{2}$ such that for each $i \in\{1,2\}$ and $v \in A_{i}$, the set $N_{H}(v) \backslash A_{i}$ is a clique. If $H$ is a linear interval graph with $A_{1}$ and $A_{2}$ on opposite ends, then $\left(H, A_{1}, A_{2}\right)$ is a linear interval strip. Now let $H$ be a directed multigraph (possibly with loops) and suppose for each edge $e$ of $H$ we have a strip $\left(H_{e}, X_{e}, Y_{e}\right)$. For each $v \in V(H)$ define

$$
C_{v}:=\left(\bigcup\left\{X_{e} \mid e \text { is directed out of } v\right\}\right) \cup\left(\bigcup\left\{Y_{e} \mid e \text { is directed into } v\right\}\right) .
$$

The graph formed by taking the disjoint union of $\left\{H_{e} \mid e \in E(H)\right\}$ and making $C_{v}$ a clique for each $v \in V(H)$ is the composition of the strips $\left(H_{e}, X_{e}, Y_{e}\right)$. Any graph formed in this way is a composition of strips. Notice that if each strip $\left(H_{e}, X_{e}, Y_{e}\right)$ in the composition has $V\left(H_{e}\right)=X_{e}=Y_{e}$, then the graph formed is just the line graph of the multigraph formed by replacing each $e \in E(H)$ with $\left|H_{e}\right|$ copies of $e$.

It is convenient to have notation and terminology for a strip together with how it attaches to the graph. An interval 2-join in a graph $G$ is an induced subgraph $H$ such that (i) $H$ is a nonempty linear interval graph, (ii) the ends of $H$ are cliques $A_{1}$ and $A_{2}$, not necessarily disjoint, (iii) $G \backslash H$ contains cliques $B_{1}, B_{2}$ (not necessarily disjoint) such that $A_{1}$ is joined to $B_{1}$ and $A_{2}$ is joined to $B_{2}$, and (iv) no other edges exist between $H$ and $G-H$. Since $A_{1}, A_{2}, B_{1}, B_{2}$ are uniquely determined by $H$, we can refer to the interval 2-join as either $H$ or, equivalently, as the quintuple $\left(H, A_{1}, A_{2}, B_{1}, B_{2}\right)$.

An interval 2-join $\left(H, A_{1}, A_{2}, B_{1}, B_{2}\right)$ is trivial if $V(H)=A_{1}=A_{2}$ and canonical if $A_{1} \cap A_{2}=\emptyset$. A canonical interval 2-join $\left(H, A_{1}, A_{2}, B_{1}, B_{2}\right)$ with leftmost vertex $v_{1}$ and rightmost vertex $v_{t}$ is reducible if $H$ is incomplete and $N_{H}\left(A_{1}\right) \backslash A_{1}=N_{H}\left(v_{1}\right) \backslash A_{1}$ or $N_{H}\left(A_{2}\right) \backslash A_{2}=N_{H}\left(v_{t}\right) \backslash A_{2}$. We call such a canonical interval 2-join reducible because we can reduce it as follows. Suppose $H$ is incomplete and $N_{H}\left(A_{1}\right) \backslash A_{1}=N_{H}\left(v_{1}\right) \backslash A_{1}$. Let $C:=N_{H}\left(v_{1}\right) \backslash A_{1}$, let $A_{1}^{\prime}:=C \backslash A_{2}$, and let $A_{2}^{\prime}:=A_{2} \backslash C$. Since $H$ is incomplete, $v_{t} \in A_{2}^{\prime}$, 
so $H^{\prime}:=G\left[A_{1}^{\prime} \cup A_{2}^{\prime}\right]$ is a nonempty linear interval graph that gives the reduced canonical interval 2-join $\left(H^{\prime}, A_{1}^{\prime}, A_{2}^{\prime}, A_{1} \cup\left(C \cap A_{2}\right), B_{2} \cup\left(C \cap A_{2}\right)\right)$.

Note that reducing an interval 2 -join yields an interval 2-join with a smaller strip. Note also that reducing a canonical interval 2-join again yields a canonical interval 2 -join. The process of reducing a 2-join allows us to refine the composition representation, and to get a representation with more strips. In particular, in a representation with the maximum number of strips, every 2 -join is irreducible.

\subsection{Coloring from Graph Orientations}

In this section, we show how we can orient the edges of a graph $G$ to prove upper bounds on $\chi_{\ell}(G)$ and $\chi_{O L}(G)$. It is well-known that if a graph $G$ is $k$-degenerate, then $\chi(G) \leq k+1$; and this upper bound holds also for $\chi_{\ell}(G)$ and $\chi_{O L}(G)$. If $v_{1}, \ldots, v_{n}$ is a vertex order such that each $v_{i}$ has at most $k$ neighbors with smaller index, then we use at most $k+1$ colors when we color greedily in order of increasing index. We can view this bound in terms of orientations as follows. Orient each edge $v_{i} v_{j}$ as $v_{i} \rightarrow v_{j}$ when $i>j$. Now $\chi(G) \leq k+1$ whenever $G$ has an acyclic orientation $D$ with maximum outdegree $k$. Alon and Tarsi proved the following far-reaching generalization, where $D$ need not be acyclic.

Theorem 1.4 (Alon-Tarsi [4]). Let $f: V \rightarrow \mathbb{Z}^{+}$be a list size assigment, and let $D$ be an orientation of $E(G)$ in which $|E E(D)| \neq|E O(D)|$, where $E E(D)$ and $E O(D)$ are the sets of spanning Eulerian subgraphs of $D$ with an even (resp. odd) number of edges. If $f(v)>d_{D}^{+}(v)$ for all $v \in V(G)$, then $G$ is $f$-choosable. (In fact, $f$-paintable.)

Now we consider the other standard technique for coloring graphs via orientations. A kernel of a digraph $D$ is an independent set $I$ such that each vertex not in $I$ has an outneighbor in $I$. A digraph is kernel-perfect if every induced subgraph has a kernel. Most applications of kernels to list-coloring use the following lemma of Bondy, Boppana, and Siegel.

Lemma 1.5 (Bondy-Boppana-Siegel [4, Remark 2.4],[14, Lemma 2.1]). Let $f: V \rightarrow \mathbb{Z}^{+}$be a list size assigment, and let $D$ be a kernel-perfect orientation of $E(G)$. If $f(v)>d_{D}^{+}(v)$ for all $v \in V(G)$, then $G$ is $f$-choosable. (In fact, $f$-paintable.)

We can easily prove Lemma 1.5 by induction. Given such an orientation, on each round Painter chooses as his independent set a kernel of the subgraph induced by the vertices listed by Lister. This technique is called the Kernel Method. Both Theorem 1.4 and Lemma 1.5 were originally proved for list coloring, and then extended to online list-coloring by Schauz [23]. (The extension of Lemma 1.5 has the same proof as the original. However, the extension of Theorem 1.4 requires significant work.)

Our proofs in this paper rely heavily on both of these techniques, so the following definitions are useful. A graph $H$ is $f-A T$ if it has an orientation $D$ with $f(v)>d_{D}^{+}(v)$ for all $v \in V(H)$ and with different numbers of even and odd spanning Eulerian subgraphs. Such a $D$ is an Alon-Tarsi orientation for $f$ and $H$. A graph $H$ is $f$-KP if some supergraph $H^{\prime}$ of $H$ has a kernel-perfect orientation where $f(v)>d^{+}(v)$ for all $v \in V\left(H^{\prime}\right)$. Allowing this supergraph for KP gives us more power. For example, $K_{4}-e$ has no kernel-perfect 
orientation showing it is degree-choosable, but if we double the edge in two triangles, then there is such an orientation. We could allow a supergraph for AT as well, but this doesn't give us any more power, as we will see in Lemma 1.6. Since our focus in this paper is the Borodin-Kostochka conjecture, we have one more definition.

Definition 1. A connected graph $G$ is $B K$-free if it does not contain an induced subgraph $H$ that is $f_{H}$-AT or $f_{H}$-KP where $f_{H}(v):=d_{H}(v)-1+\Delta(G)-d_{G}(v)$ for all $v \in V(H)$.

The motivation for this definition is that any minimal counterexample to Theorem 1.2 must be BK-free. To see this for list-coloring is easy. Suppose $G$ is not BK-free; say it contains subgraph $H$ that is $f$-AT or $f$-KP. By minimality, color $G \backslash H$. Now, by definition, we can extend the coloring to $H$. The same idea works for online list coloring. On each round, Painter first plays optimally on $G \backslash H$, then plays optimally on $H$ (omitting from $H$ any vertices with neighbors in $G$ that Painter chose on that round). So in particular, if $G$ is BK-free, then $\delta(G) \geq \Delta(G)-1$. Thus, a vertex $v$ is high if $d(v)=\Delta(G)$ and low if $d(v)=\Delta(G)-1$. When we write that subgraph $H$ is $f$-AT or $f$-KP without specifying $f$, we mean $f(v)=d_{H}(v)-1+\Delta(G)-d_{G}(v)$ (so $f(v)=d_{H}(v)-1$ when $v$ is high in $G$ and $f(v)=d_{H}(v)$ when $v$ is low $)$.

A special case of the weak perfect graph theorem states that if $G$ is the complement of a bipartite graph, then $\chi(G)=\omega(G)$. In this section, we prove a strengthening of the analogous statement for Alon-Tarsi orientations. This result plays a key role in Section 2.2, where we handle non-linear homogeneous pairs of cliques.

It is well known that for a graph $G$, if $H \subseteq G$, then $\chi(H) \leq \chi(G)$ and $\chi_{\ell}(H) \leq \chi_{\ell}(G)$. More generally, if $f$ is a list-size assignment and $G$ is $f$-choosable or $f$-paintable, then so is $H$. It is natural to expect that an analogous statement holds for Alon-Tarsi orientations. Indeed it does, as we show in Lemma 1.6.

Given a graph $G$, let $v_{1}, \ldots, v_{n}$ be an arbitrary ordering of $V(G)$. The graph polynomial, $g$, of $G$ is given by $g=\prod_{v_{i} v_{j} \in E(G), i<j}\left(x_{i}-x_{j}\right)$. Note that $g$ is independent of the ordering of $V(G)$, up to a factor of \pm 1 . For a polynomial $g \in \mathbb{F}\left[x_{1}, \ldots, x_{n}\right]$, we write $g_{k_{1}, \ldots, k_{n}}$ for the coefficient in $g$ of $x_{1}^{k_{1}} \cdots x_{n}^{k_{n}}$. Alon and Tarsi [4, Corollary 2.3] observed that $G$ is $f$-AT precisely when there exist $k_{i}$ such that $f\left(v_{i}\right) \geq k_{i}+1$ for all $i$ and the graph polynomial has $g_{k_{1}, \ldots, k_{n}} \neq 0$.

Lemma 1.6. If a graph $G$ is $f$-AT (for any particular function $f$ ) and $e \in E(G)$, then $G-e$ is also $f$-AT. More generally, if $H$ is a subgraph of $G$ and $G$ is $f$-AT, then so is $H$.

Proof. The second statement follows from the first by induction on $|E(G) \backslash E(H)|$. If $|V(H)|<|V(G)|$, then for each vertex in $V(G) \backslash V(H)$, we first delete all of its incident edges. Now adding or removing an isolated vertex $v$ has no effect on the graph polynomial.

Fix a graph $G$ and a function $f$ such that $G$ is $f$-AT. As noted above, $G$ is $f$-AT if and only if the graph polynomial of $G$ has a nonzero term $x_{1}^{k_{1}} \cdots x_{n}^{k_{n}}$, where $x_{i}$ is the variable corresponding to vertex $v_{i}$, such that $f\left(v_{i}\right)>k_{i}$ for all $i$.

Suppose the lemma is false, that is, there exists $e \in E(G)$ such that $G-e$ is not $f$-AT. Let $v_{1}$ and $v_{2}$ denote the endpoints of $e$. Since $G-e$ is not $f$-AT, its graph polynomial has no nonzero term as above. That is, for every term $x_{1}^{j_{1}} \cdots x_{n}^{j_{n}}$, there exists $i$ such that $f\left(v_{i}\right) \leq j_{i}$. 
Now the graph polynomial of $G$ is formed from that of $G-e$ by multiplying by $\left(x_{1}-x_{2}\right)$. Terms may cancel, but the exponents never go down. Since $G-e$ is not $f$-AT, for every term $x_{1}^{j_{1}} \cdots x_{n}^{j_{n}}$ in the polynomial of $G-e$, there exists $i$ such that $f\left(v_{i}\right) \leq j_{i}$. Thus, for any remaining term $x_{1}^{k_{1}} \cdots x_{n}^{k_{n}}$ in the graph polynomial of $G$, there exists $i$ such that $f\left(v_{i}\right) \leq k_{i}$; in particular, we have $f\left(v_{i}\right) \leq j_{i} \leq k_{i}$, where $i$ is chosen to show that $G-e$ is not $f$-AT. Hence, $G$ is not $f$-AT, a contradiction.

Let $K_{2 * t}$ denote the complete multipartite graph with $t$ parts of size 2. Both [15] and [17] showed that $K_{2 * t}$ is $f$-AT when $f(v)=t$ for all $v$. So a direct application of Lemma 1.6 yields the following.

Corollary 1.7. If $G \subseteq K_{2 * t}$, then $G$ is $f$-AT when $f(v)=t$ for all $v$. So, if $G$ is BK-free,

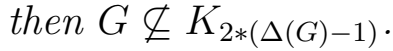

We need a refinement of Corollary 1.7 that works for $G \subseteq K_{s} \vee K_{2 * t}$ when some of the lists are smaller than size $s+t$. The idea used to prove Theorem [1.4 was generalized [2, 3] to what is now called the Combinatorial Nullstellensatz. Schauz [22] further sharpened this result, by proving the following coefficient formula. Versions of this sharper result were also proved by Hefetz [15] and Lasoń [20]. Our presentation follows Lasoń. Recall that for a polynomial $g \in \mathbb{F}\left[x_{1}, \ldots, x_{n}\right]$, we write $g_{k_{1}, \ldots, k_{n}}$ for the coefficient in $g$ of $x_{1}^{k_{1}} \cdots x_{n}^{k_{n}}$.

Lemma 1.8 (Schauz [22]). Suppose $g \in \mathbb{F}\left[x_{1}, \ldots, x_{n}\right]$ and $k_{1}, \ldots, k_{n} \in \mathbb{N}$ with $\sum_{i \in[n]} k_{i}=$ $\operatorname{deg}(g)$. For any $C_{1}, \ldots, C_{n} \subseteq \mathbb{F}$ with $\left|C_{i}\right|=k_{i}+1$, we have

$$
g_{k_{1}, \ldots, k_{n}}=\sum_{\left(c_{1}, \ldots, c_{n}\right) \in C_{1} \times \cdots \times C_{n}} \frac{f\left(c_{1}, \ldots, c_{n}\right)}{N\left(c_{1}, \ldots, c_{n}\right)},
$$

where

$$
N\left(c_{1}, \ldots, c_{n}\right):=\prod_{i \in[n]} \prod_{d \in C_{i}-c_{i}}\left(c_{i}-d\right) .
$$

Now we use Lemma 1.8 to prove the desired strengthening of Corollary 1.7

Lemma 1.9. Let $G=K_{s} \vee K_{2 * t}$, let $A$ be an $(s+t)$-clique in $G$, and let $B=V(G) \backslash A$. Now $G$ is $f$-AT whenever $f(v) \geq s+t$ for all $v \in A$ and $f(v) \geq t$ for all $v \in B$.

Proof. Let $r=s+t$. Say $A=\left\{a_{1}, \ldots, a_{r}\right\}$ and $B=\left\{b_{1}, \ldots, b_{t}\right\}$. Let $g$ be the graph polynomial of $G$, with the vertex order $a_{1}, b_{1}, \ldots, a_{t}, b_{t}, a_{t+1}, \ldots, a_{r}$. Recall that it suffices to show that $g_{k_{1}, \ldots, k_{n}} \neq 0$ for some choice of $k_{1}, \ldots, k_{n}$ with $f\left(v_{i}\right)>k_{i}$ for all $i$. To do this, we apply Lemma 1.8.

Now $\operatorname{deg}(g)=|E(G)|=\left(\begin{array}{l}r \\ 2\end{array}\right)+\left(\begin{array}{l}t \\ 2\end{array}\right)+t(r-1)$. For each $i \in[t]$, let $L\left(a_{i}\right)=[r]$ and $L\left(b_{i}\right)=[t]$. For each $i \in[r] \backslash[t]$, let $L\left(a_{i}\right)=[i]$. Note that $\sum_{i \in[r]}\left(\left|L\left(a_{i}\right)\right|-1\right)+\sum_{i \in[t]}\left(\left|L\left(b_{i}\right)\right|-1\right)=$ $t(r-1)+(r-t)(t+r-1) / 2+t(t-1)=|E(G)|$, so these lists will work for the $A_{j}$ in Lemma 1.8. Also note that $\left|L\left(a_{i}\right)\right| \leq f\left(a_{i}\right)$ for all $i \in[r]$ and $\left|L\left(b_{i}\right)\right| \leq f\left(b_{i}\right)$ for all $i \in[t]$, so showing that the corresponding coefficient of $g$ is nonzero will prove the lemma.

The sum in (11) of Lemma 1.8 is zero at every term that is not a proper coloring of $G$ from $L$. By construction, all proper colorings of $G$ from $L$ must assign $1, \ldots, t$ to vertices of $B$. 
For each vertex $b_{i}$ of $B$, its only non-neighbor is $a_{i}$. Hence in every proper coloring of $G$ from $L$, each of colors $1, \ldots, t$ is assigned to some pair $\left(a_{i}, b_{i}\right)$. As a result, vertex $a_{t+1}$ must get color $t+1$, vertex $a_{t+2}$ must get color $t+2$, etc. More precisely, for every $i \in\{t+1, \ldots, r\}$, vertex $a_{i}$ gets color $i$. Said differently, any coloring of $G$ from $L$ can be obtained from any given such coloring by permuting $1, \ldots, t$. Thus, the function $N$ in (2) of Lemma 1.8 gives the same nonzero value on all such colorings (since for all $i, j \in[t]$, we have $L\left(a_{i}\right)=L\left(a_{j}\right)$ and $\left.L\left(b_{i}\right)=L\left(b_{j}\right)\right)$.

The previous paragraph implies that the sum in (1) of Lemma 1.8 is a nonzero constant multiplied by the sum of the graph polynomial $g^{\prime}$ of $G\left[a_{1}, \ldots, a_{t}, b_{1}, \ldots, b_{t}\right]$ evaluated at some points where $a_{i}$ and $b_{i}$ get the same value for each $i \in[t]$. Any such evaluation is the fourth power of an integer, since edges $a_{i} a_{j}, a_{i} b_{j}, b_{i} a_{j}, b_{i} b_{j}$ each contribute the same factor. In particular, all the terms in the sum have the same sign. Hence, by Lemma 1.8, the coefficient in question is nonzero, so $G$ is $f$-AT.

Lemma 1.10. Let $G$ be the complement of a bipartite graph with parts $A$ and $B$. If $f(v) \geq$ $\omega(G)$ for all $v \in A$ and $f(v) \geq|B|$ for all $v \in B$, then $G$ is $f$-AT.

Proof. Define $G$ and $f$ as in the lemma. We can assume that $|A|=\omega(G)$; so, in particular, $|A| \geq|B|$. If not, then add $\omega(G)-|A|$ vertices to $A$ that are adjacent only to vertices in $A$ (this does not increase $f(v)$ for any $v$ ). So we have $|B| \leq|A|=\omega(G)$. For each $S \subseteq A \cup B$, let $\bar{N}(S)$ denote the neighbors of at least one vertex of $S$ in $\bar{G}$, the complement of $G$. Since $\omega(G)=|A|$, for each $S \subseteq B$, we have $|\bar{N}(S)| \geq|S|$; otherwise $A \cup S \backslash \bar{N}(S)$ is a clique of $G$ bigger than $A$. So Hall's Theorem implies that $G \subseteq K_{|A|-|B|} \vee K_{2 *|B|}$. Hence, by Lemma 1.9 and Lemma 1.6, $G$ is $f$-AT.

\section{Reduction from Quasi-line Graphs to Line Graphs}

In this section, we prove that Theorem 1.2 is true (for quasi-line graphs) if it is true for line graphs. Recall our general approach, based on the quasi-line structure theorem, given in Theorem 1.3. We assume that Theorem 1.2 is false, and choose $G$ to be a minimal counterexample; thus, $G$ is BK-free. In Section 2.1, we prove that $G$ is not a circular interval graph. In Section 2.2, we prove that $G$ has no non-linear homogeneous pair of cliques. Finally, in Section 2.3, we consider when $G$ is a composition of linear interval strips (which include line graphs, as a special case). We reduce this case to the case of line graphs, which we handle in Section 3 .

\subsection{Handling circular interval graphs}

The following proof is nearly identical to the one we gave in [10] for the list-coloring analogue, but we reproduce it here for completeness. One notable difference is that all of the listcoloring lemmas used to show reducibility in that proof have been replaced here by the Alon-Tarsi orientations in Figure 1.

Lemma 2.1. Let $G$ be a BK-free graph with $\omega(G)<\Delta(G)$. If $G$ is a circular interval graph, then $\Delta(G)<9$. 
Proof. Suppose to the contrary that $G$ is a circular interval graph that is BK-free, has $\Delta(G) \geq 9$, and does not contain $K_{\Delta(G)}$. Let $K$ be a maximum clique in $G$. By symmetry we may assume that $V(K)=\left\{v_{1}, v_{2}, \ldots, v_{t}\right\}$ for some $t \leq \Delta-1$; further, if possible we label the vertices so that $v_{t-3} \leftrightarrow v_{t+1}$ and the edge goes through $v_{t-2}, v_{t-1}, v_{t}$.

Claim 1. $v_{1} \leftrightarrow v_{t+1}$ and $v_{2} \leftrightarrow v_{t+2}$ and $v_{1} \leftrightarrow v_{t+2}$. Assume the contrary. Clearly we cannot have $v_{1} \leftrightarrow v_{t+1}$ and have the edge go through $v_{2}, v_{3}, \ldots, v_{t}$ (since then we get a clique of size $t+1)$. Similarly, we cannot have $v_{2} \leftrightarrow v_{t+2}$ and have the edge go through $v_{3}, v_{4}, \ldots, v_{t+1}$. So assume the edge $v_{1} v_{t+2}$ exists and goes around the other way. If $v_{1} \leftrightarrow$ $v_{t+1}$, then let $G^{\prime}=G \backslash\left\{v_{1}\right\}$ and if $v_{1} \leftrightarrow v_{t+1}$, then let $G^{\prime}=G \backslash\left\{v_{1}, v_{t+1}\right\}$. Now let $V_{1}=\left\{v_{2}, v_{3}, \ldots, v_{t}\right\}$ and $V_{2}=V\left(G^{\prime}\right) \backslash V_{1}$. Let $K^{\prime}=G\left[V_{1}\right]$ and $L^{\prime}=G\left[V_{2}\right]$; note that $K^{\prime}$ and $L^{\prime}$ are each cliques of size at most $\Delta-2$. Now for each $S \subseteq V_{2}$, we have $\left|N_{\bar{G}}(S) \cap V_{1}\right| \geq|S|$ (otherwise we get a clique of size $t$ in $G^{\prime}$ and a clique of size $t+1$ in $G$ ). Now by Hall's Theorem, we have a matching in $\bar{G}$ between $V_{1}$ and $V_{2}$ that saturates $V_{2}$. This implies that $G^{\prime} \subseteq K_{2 *(\Delta-2)}$, which in turn gives $G \subseteq K_{2 *(\Delta-1)}$. This contradicts Corollary 1.7 .

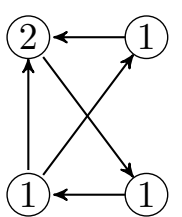

(a) : $\mathrm{EE}=2, \mathrm{EO}=1$

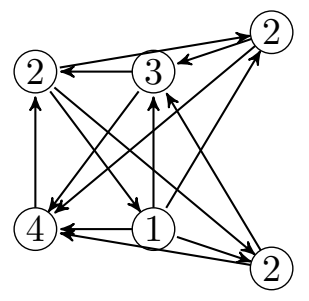

(d) $\mathrm{EE}=16, \mathrm{EO}=17$

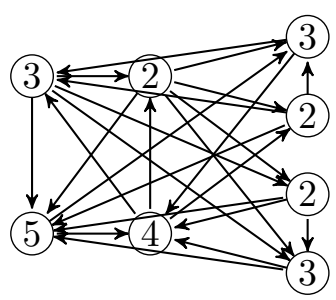

(g) $\mathrm{EE}=1097, \mathrm{EO}=1096$

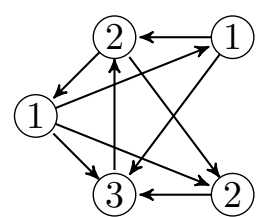

(b) $\mathrm{EE}=4, \mathrm{EO}=3$

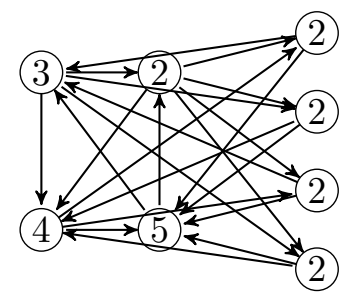

(e) $\mathrm{EE}=512, \mathrm{EO}=515$

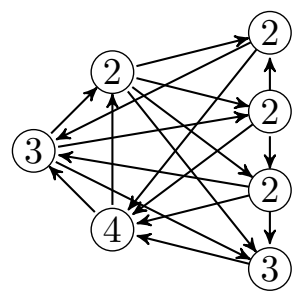

(h) $\mathrm{EE}=108, \mathrm{EO}=107$

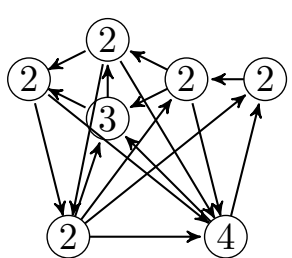

(c) $\mathrm{EE}=81, \mathrm{EO}=80$

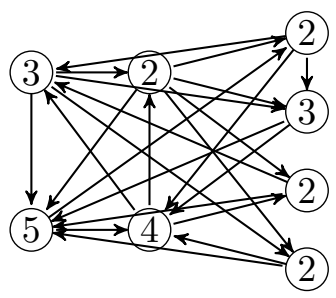

(f) $\mathrm{EE}=751, \mathrm{EO}=750$

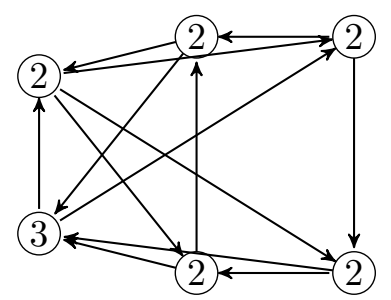

(i) $: \mathrm{EE}=30, \mathrm{EO}=28$

Figure 1: Subgraphs forbidden by Alon-Tarsi orientations, used in Lemma 2.1.

Claim 2. $v_{t-3} \leftrightarrow v_{t+1}$ and the edge passes through $v_{t-2}, v_{t-1}, v_{t}$. Assume the contrary. Since $t \leq \Delta-1$ and $\delta(G) \geq \Delta-1$, each vertex in $K$ has a neighbor outside of $K$; in particular, $v_{4}$ has some neighbor outside of $K$. If $t \geq 7$, then by (reflectional) symmetry we could have labeled the vertices so that $v_{t-3} \leftrightarrow v_{t+1}$ (and the edge passes through $v_{t-2}, v_{t-1}, v_{t}$ ). So we must have $t \leq 6$. Each vertex $v$ that is high has either at least $\lceil\Delta / 2\rceil$ clockwise neighbors or at least $\lceil\Delta / 2\rceil$ counterclockwise neighbors. This gives a clique of size $1+\lceil\Delta / 2\rceil \geq 6$. 
Thus, $t=6$ and $v_{t-3}=v_{3}$. If $v_{3}$ is high, then either $v_{3}$ has at least 4 clockwise neighbors, so $v_{3} \leftrightarrow v_{7}$, or else $v_{3}$ has at least 6 counterclockwise neighbors, so $|K| \geq 7$. Thus, we may assume that $v_{3}$ is low; by symmetry (and our choice of labeling prior to Claim 1 ) $v_{4}$ is also low. Now since $v_{4}$ has only 3 counterclockwise neighbors, we get $v_{4} \leftrightarrow v_{7}$ (in fact, we get $\left.v_{4} \leftrightarrow v_{9}\right)$. Thus, $\left\{v_{3}, v_{4}, v_{5}, v_{6}, v_{7}\right\}$ induces $K_{3} \vee E_{2}$ with a low degree vertex in both the $K_{3}$ and the $E_{2}$, which is $f$-AT, as shown in Figure 1 1 (b).

Claim 3. $v_{t-2} \leftrightarrow v_{t+2}$ and $v_{t-1} \leftrightarrow v_{t+2}$. First, assume to the contrary that $v_{t-2} \leftrightarrow$ $v_{t+2}$. By Claim 1 the edge must go through $v_{t-1}, v_{t}, v_{t+1}$. If $v_{t-3} \leftrightarrow v_{t+2}$, then the set $\left\{v_{1}, v_{2}, v_{t-3}, v_{t-2}, v_{t-1}, v_{t}, v_{t+1}, v_{t+2}\right\}$ induces $K_{4} \vee B$, where $B$ is not almost complete; this subgraph is $f$-AT, as shown in Figure1(e) (i). If $v_{t-3} \leftrightarrow v_{t+2}$, then the set $\left\{v_{1}, v_{t-3}, v_{t-2}, v_{t-1}\right.$, $\left.v_{t}, v_{t+1}, v_{t+2}\right\}$ induces $K_{3} \vee P_{4}$ is $f$-AT, as shown in Figure 1(h). Hence, $v_{t-2} \nleftarrow v_{t+2}$.

So assume that $v_{t-1} \leftrightarrow v_{t+2}$. Now $\left\{v_{1}, v_{t-3}, v_{t-2}, v_{t-1}, v_{t}, v_{t+1}, v_{t+2}\right\}$ induces $K_{2} \vee$ antichair (with $v_{t-1}, v_{t}$ in the $K_{2}$ ), which is $f$-AT, as shown in Figure 1. (c).

Claim 4. The lemma is true. Let $S=\left\{v_{t-3}, v_{t-2}, v_{t-1}, v_{t}\right\}$. If any vertex of $S$ is low, then $S \cup\left\{v_{1}, v_{t+1}\right\}$ induces $K_{4} \vee E_{2}$ with a low vertex in the $K_{4}$, which is $f$-AT, as shown in Figure [1](d), So all of $S$ is high. If $v_{t} \leftrightarrow v_{t+2}$, then $\left\{v_{t}, v_{t-1}, \ldots, v_{t-\Delta+1}\right\}$ (subscripts are modulo $n$ ) induces $K_{\Delta}$. So $v_{t} \leftrightarrow v_{t+2}$. Since $v_{t-1} \leftrightarrow v_{t+2}$ and all of $S$ is high, there exists a vertex $v_{n}$ that is not adjacent to $v_{t}$ but is adjacent to the rest of $S$. Formally, $v_{n} \in\left(\cap_{v \in\left(S \backslash\left\{v_{t}\right\}\right)} N(v)\right) \backslash N\left(v_{t}\right)$. Clearly the edge from $v_{t-1}$ to $v_{n}$ must go through $v_{t-2}$. Since $v_{n} \leftrightarrow v_{t}$, we have $n<1$. However, if $n<0$, then $G$ contains a clique larger than $K$. Thus, we may assume $v_{n}=v_{0}$.

If $v_{n} \leftrightarrow v_{t+1}$, then $G$ can be covered by two cliques: $K$ and $G \backslash K$. As in Claim 1 , we show that $\bar{G}$ has a matching between $K$ and $G \backslash K$ that saturates $G \backslash K$. Thus, $G \subseteq K_{2 *(\Delta-1)}$, which contradicts Corollary [1.7. Since $v_{n} \leftrightarrow v_{t+1}$, we get $K_{3} \vee P_{4}$ induced by $\left\{v_{t+1}, v_{t}, v_{t-1}, v_{t-2}, v_{t-3}, v_{1}, v_{n}\right\}$. Again, this subgraph is $f$-AT, as shown in Figure 1(h).

\subsection{Handling non-linear homogeneous pairs of cliques}

Lemma 2.2. Let $G$ be a BK-free graph with $\omega(G)<\Delta(G)$. If $G$ has a non-linear homogeneous pair of cliques, then $\Delta(G)<9$.

Proof. Suppose to the contrary that $G$ is a BK-free graph with $\Delta(G) \geq 9$ and $\omega(G)<\Delta(G)$ and that $G$ contains a non-linear homogeneous pair of cliques $(A, B)$. Let $H:=G[A \cup B]$ and let $f_{H}(v):=d_{H}(v)-1+\Delta(G)-d_{G}(v)$ for all $v \in V(H)$. Now $H$ is not complete, since it it is non-linear and hence induces a $C_{4}$. Our general approach is to show that $G$ contains some induced $f$-AT subgraph in Figure 3 , where $f(v)=d(v)$ when $v$ is low and $f(v)=d(v)-1$ otherwise.

Note that $f_{H}(v)=d_{H}(v)-1$ if $v$ is high and $f_{H}(v)=d_{H}(v)$ if $v$ is low. For each $X \in\{A, B\}$, let $\delta_{X}:=\min _{v \in X} d_{H}(v)$ and $\Delta_{X}:=\max _{v \in X} d_{H}(v)$. Since each vertex in $G$ has degree either $\Delta(G)$ or $\Delta(G)-1$, and $(A, B)$ is a homogeneous pair of cliques, we have $\Delta_{X} \leq \delta_{X}+1$ for each $X \in\{A, B\}$; equality holds when $X$ contains both a high and a low vertex. Also, $f_{H}(v)=\Delta_{X}-1$ for each $v \in X$ whenever $X$ contains a high vertex. Let $W$ be an arbitrary maximum clique in $H$.

Claim 0. For $\{X, Y\}=\{A, B\}$, either $\Delta_{X} \leq|W|$ or $\Delta_{Y} \leq|Y|$. Since $G$ is BK-free, $f_{H}$ cannot satisfy the hypotheses of Lemma 1.10. Unpacking what that means gives precisely 
$\Delta_{X} \leq|W|$ or $\Delta_{Y} \leq|Y|$.

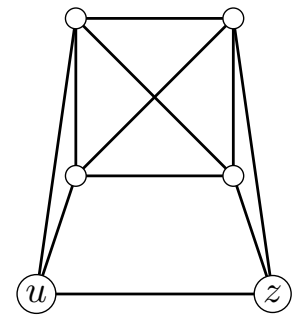

(a) 3 and 3

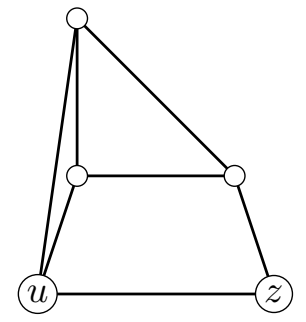

(b) 3 and 2

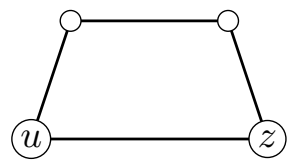

(c) 2 and 2

Figure 2: Possible non-linear homogeneous pair of cliques.

Claim 1. Either $|W \cap A| \leq 1$ or $|W \cap B| \leq 1$. Suppose instead that $|W \cap X| \geq 2$ for all $X \in\{A, B\}$. Now $\Delta_{X} \geq|X|+1$ for all $X \in\{A, B\}$, so applying Claim 0 gives $\Delta_{X} \leq|W|$ for all $X \in\{A, B\}$. For all $v \in W \cap X$, we have $d_{H}(v) \geq|W|-1+|X \backslash W|$. Hence $|W| \geq \Delta_{X} \geq|W|-1+|X \backslash W|$, which gives $|X \cap W| \geq|X|-1$ for all $X \in\{A, B\}$.

Now we show that $A \subseteq W$ or $B \subseteq W$. Suppose instead that $|W \cap A|=|A|-1$ and $|W \cap B|=|B|-1$. Let $\{u\}=A \backslash W$ and $\{z\}=B \backslash W$. If $u$ has a neighbor $v \in W \cap B$, then $d_{H}(v) \geq|W|+1$, a contradiction. Similarly, $z$ has no neighbors in $W \cap A$. So $d_{A}(z) \leq 1$ and $d_{B}(u) \leq 1$, which implies that $\Delta_{A} \leq|A|+1$ and $\Delta_{B} \leq|B|+1$. Now $|B|+1 \geq \Delta_{B} \geq|B|-1+|W \cap A|$, so $|W \cap A| \leq 2$. By assumption $|W \cap A|=|A|-1$, so $|A|=|W \cap A|+1 \leq 3$; similarly, $|B| \leq 3$.

Recall that $|W \cap A| \geq 2,|W \cap B| \geq 2,|A| \leq 3$, and $|B| \leq 3$. Now our assumption that $|W \cap A|=|A|-1$ and $|W \cap B|=|B|-1$ gives $|A|=|B|=3$. So $H$ must be as in Figure 2[(a). Hence $d_{G}(u)=d_{G}(z)=\Delta(G)-1$. But now either (i) some vertex outside $H$ is joined to just one side of $H$ and $G$ contains the forbidden induced subgraph in Figure 3.(a) or (ii) some vertex outside $H$ is joined to both sides of $H$ and $G$ contains the graph in Figure 3)(b). Each of these induced subgraphs is forbidden, which gives a contradiction. Thus, $A \subseteq W$ or $B \subseteq W$.

By symmetry, suppose $A \subseteq W$. Now we get $|W| \geq|A|+|B \cap W| \geq|A|+(|B|-1)$. Thus, $H$ is almost complete, so it does not contain an induced $C_{4}$; this contradicts the hypothesis of the lemma and so proves the claim.

Claim 2. Either $\omega(H) \leq 2$ or the only possible maximum cliques in $H$ are $A$ and $B$. Suppose to the contrary that $\omega(H) \geq 3$ and that $W$ is a maximum clique in $H$ with $|W \cap A| \geq 1$ and $|W \cap B| \geq 1$. By Claim 1 and symmetry, we may assume $|W \cap B|=1$. Let $z_{1}, \ldots, z_{t}$ be the vertices of $B \backslash W$. Since $|W|=\omega(H) \geq 3$ and $|W \cap B|=1$, we must have $|W \cap A| \geq 2$ and hence $|W \cap A| \geq|A|-1$, as in the first paragraph of the proof of Claim 1.

So we have the two cases (i) $A \subseteq W$ and (ii) $|A \cap W|=|A|-1$. First suppose that $A \subseteq W$. We begin with the case $|A|=2$. Since $|W \cap B|=1$ and $3 \leq|W| \leq|A|+|W \cap B| \leq 3$, we get that $|B| \leq|W| \leq 3$. We must have $|B| \geq 3$, since otherwise $H$ is almost complete, so it cannot induce $C_{4}$. So $|B|=3$, and $\Delta_{B} \geq|W|+1$. Since $\Delta_{B}-\delta_{B} \leq 1$, each of $z_{1}$ and $z_{2}$ has a neighbor in $A$; thus $\Delta_{A} \geq|A|+1$, which contradicts Claim 0. Hence, $|A| \geq 3$.

Suppose now that $A \subseteq W$ and $|A| \geq 3$. Since $\Delta_{B}-\delta_{B} \leq 1$, each $z_{i}$ is nonadjacent to exactly one vertex of $A$; call it $u_{i}$. By Claim 0, no vertex in $A$ can have two neighbors outside 


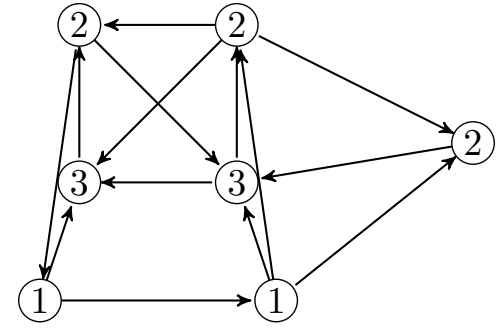

(a) $\mathrm{E}=14, \mathrm{O}=12$

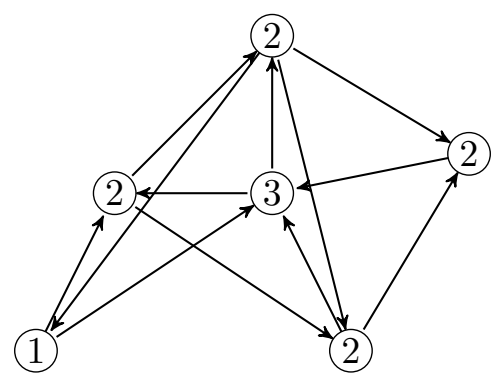

(d) $\mathrm{EE}=14, \mathrm{EO}=15$

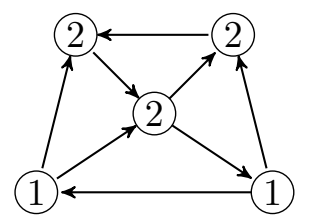

(b) $\mathrm{E}=4, \mathrm{O}=2$

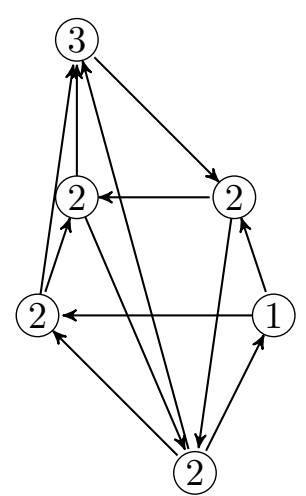

(e) $\mathrm{EE}=13, \mathrm{EO}=11$

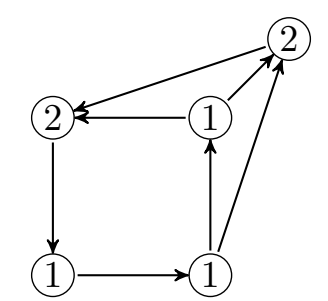

(c) $\mathrm{EE}=3, \mathrm{EO}=1$

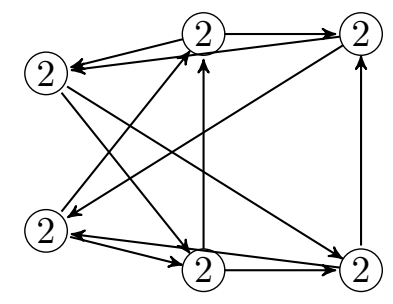

(g) $: \mathrm{EE}=22, \mathrm{EO}=16$

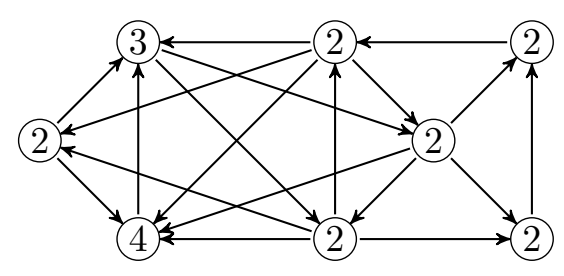

(h) $\mathrm{EE}=72, \mathrm{EO}=74$

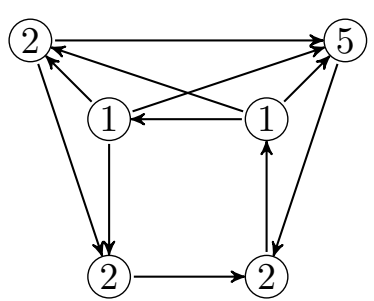

(f) $\mathrm{EE}=5, \mathrm{EO}=3$

Figure 3: Subgraphs forbidden by Alon-Tarsi orientations, used in Lemma 2.2. 
of $W$; so $t=1$ and hence $|B|=2$. Now again, $|W| \geq|A|+|B|-1$, so $H$ cannot induce a $C_{4}$; this contradicts the hypothesis of the lemma. Thus, $A \nsubseteq W$.

So assume instead that $|A \cap W|=|A|-1$. Note that $|A| \geq 3$, since $|A \cap W| \geq 2$.

Let $\{u\}=A \backslash W$ and $\{w\}=B \cap W$. Note that $u$ is not adjacent to $w$, since $u \notin W$. Since $|W \cap A| \geq 2$, Claim 0 implies that $\Delta_{A} \leq|W|$. Hence each $v \in A \cap W$ has no neighbors in $B-w$. Also $w$ has at least two neighbors in $A$, so $\delta_{B} \geq|B|$. Now $\Delta_{B}-\delta_{B} \leq 1$ implies that $|A| \leq 3$. Since also $|A| \geq 3$, we have $|A|=3$ and each vertex of $W \backslash B$ is adjacent to $u$. Now $|A|=|W| \geq \Delta_{A} \geq d_{H}(u)=|A|-1+t$, so $t \leq 1$. Actually $t=1$, since otherwise $H$ is almost complete, so it cannot induce $C_{4}$.

Now $H$ must be as in Figure 2(b), with $z$ low. If some vertex outside of $H$ is adjacent to all of $H$, then $G$ contains the $f$-AT subgraph in Figure 3) a contradiction. So each neighbor of $A$ outside of $H$ is adjacent to $A$ and not adjacent to $B$. Since $G$ is quasi-line and none of its neighbors outside $H$ is adjacent to $B$, all of these outside neighbors form a clique. If some vertex of $A$ is high, then these outside neighbors, together with $A$, form a $K_{\Delta(G)}$, which is a contradiction. Otherwise, all of $A$ is low. In this case, $G$ contains the reducible configuration in Figure 3 (c).

By symmetry, we henceforth assume that $|A| \geq|B|$.

Claim 3. $A$ is a maximum clique in $H$ and $\Delta_{A} \leq|A|$. First, suppose $\omega(H) \leq 2$. Recall that $H$ induces a $C_{4}$, so $|A| \geq 2$. In fact, we must have $H=C_{4}$, since $2 \geq \omega(H) \geq|A| \geq$ $|B| \geq 2$. Hence, the degree condition is satisfied.

Now assume $\omega(H)>2$. By Claim 2, no maximum clique in $H$ has vertices in both $A$ and $B$. In particular, $A$ is a maximum clique. If $\Delta_{A}>|A|$, then there exists $v \in A$ with at least two neighbors in $B$. Since $\Delta_{A}-\delta_{A} \leq 1$, each other vertex in $A$ has at least one neighbor in $B$. Now, since $|A| \geq|B|$, some vertex in $B$ has at least two neighbors in $A$. However, now we get $\Delta_{A}>|A|$ and $\Delta_{B}>|B|$, which contradicts Claim 0. Thus, the degree condition holds.

Claim 4. If all of $A$ is low, then the lemma is true. First suppose that $|A|=|B|$. If all of $B$ is low, then we have an induced $C_{4}$ in the low vertex subgraph, which is $f$-AT. So suppose that some vertex $b \in B$ is high. Since $|A|=|B|$, Claim 3 also shows that $\Delta_{B} \leq|B|$. Hence, each vertex in $B$ has at most one neighbor in $A$. So $b$ must have $\Delta-1$ neighbors in $G \backslash A$. If all neighbors of $b$ in $G \backslash H$ induce a clique, then $G$ contains a copy of $K_{\Delta(G)}$, which contradicts that $\omega(G)<\Delta(G)$. So $b$ has nonadjacent neighbors $u_{1}, u_{2}$ in $G \backslash H$. Since $G$ is quasi-line, at least one of $u_{1}$ and $u_{2}$ is complete to $A$; by symmetry, say this is $u_{1}$. Now consider an induced $C_{4}$ in $H$, together with $u_{1}$. Since all of $A$ is low, this is the configuration shown in Figure 3.(b), which is $f$-AT.

So assume instead that $|A|>|B|$. Since $\Delta_{A} \leq|A|$ (and all of $A$ is low), each vertex of $A$ must have exactly one neighbor in $B$. Since $\Delta_{B}-\delta_{B} \leq 1$, for some integer $k$, each vertex in $B$ has either $k$ or $k+1$ neighbors in $A$. Since $|A|>|B|$, we have $k \geq 1$. If there exist $b_{1}, b_{2} \in B$ each with at least two neighbors in $A$, then we have the configuration in Figure [3] vertex. If $B$ has at least two low vertices, then we have an induced $C_{4}$ of low vertices, which is $f$-AT, a contradiction. So $B$ must contain exactly one high vertex and one low vertex. Now we have the configuration in Figure 3. (c), which is $f$-AT.

Claim 5. There exists a unique vertex $w$ that is joined to all of $H$. Since $\Delta_{A} \leq|A|$, each vertex of $A$ has at most one neighbor in $B$. Since $A$ is not all low, $A \cup N(A) \backslash H$ has 
$\Delta$ vertices. Since $G$ does not contain $K_{\Delta}$, some pair of neighbors of $A$ in $G \backslash H$ must be nonadjacent. Since $G$ is quasi-line, one of those neighbors is joined to $H$; call this vertex $w$. If two vertices outside $H$ are joined to $H$, then $G$ contains the $f$-AT configuration in Figure 1[(i) or Figure 3(g). Thus, $w$ is unique.

If $|A| \geq 4$, then $G$ contains the $f$-AT subgraph in Figure $3(\mathrm{~h})$. So assume $|A| \leq 3$. Suppose $\Delta_{B}>|B|$. Since $3 \geq|A| \geq|B| \geq 2$ (and $\Delta_{A} \leq|A|$ ), we have $|A|=3$ and $|B|=2$. Now $G$ contains the $f$-AT subgraph in Figure 3 (e) or Figure [3] (if the two vertices in $B$ have a common neighbor in $A)$. So we conclude that $\Delta_{B} \leq|B|$.

If all of $B$ is low, then $G$ contains the $f$-AT subgraph in Figure [3)(b). So instead $B$ contains some high vertex $b$. Since $\Delta_{B} \leq|B|$, and $b$ is high, $B \cup N(\bar{B}) \backslash H$ contains $\Delta$ vertices. Since $G$ contains no $K_{\Delta}$, the set $N(B) \backslash H$ contains some nonneighbor of $w$. If $|B \cup(N(B) \backslash(H \cup\{w\}))| \geq 4$, then $G$ contains the $f$-AT subgraph in Figure 3 (h). The same is true if $|A \cup(N(A) \backslash(H \cup\{w\}))| \geq 4$. Since $G$ is quasi-line, $N(w)$ is contained in $H \cup N(H)=A \cup B \cup N(A) \cup N(B)$. This gives $d(w) \leq 3+3$, which contradicts that $\delta(G) \geq \Delta(G)-1 \geq 8$. This contradiction finishes the proof of the lemma.

\subsection{Handling 2-joins}

Our goal in this section is to write $G$ as a composition of linear interval strips, where each strip is complete or complete less an edge (since this implies that $G$ is very nearly a line graph). Our main tool is the following lemma, which we will apply to each interval 2-join in the representation.

Lemma 2.3. Let $G$ be a $B K$-free graph with $\Delta(G) \geq 9$ and $\omega(G)<\Delta(G)$. If $\left(H, A_{1}, A_{2}, B_{1}, B_{2}\right)$ is an irreducible canonical interval 2-join in $G$, then

(1) $B_{1} \cap B_{2}=\emptyset ;$ and,

(2) $\left|A_{1}\right|,\left|A_{2}\right| \leq 3$; and,

(3) either $H$ is complete, or $H=K_{|H|}-x y$ and $|H| \leq 6$, where $x$ and $y$ are low in $G$.

Proof. The most interesting of the three conclusions in the lemma is (3). If $H$ is complete, for every choice of $H$, then $G$ is a line graph, which we handle in Section 3, So (3) proves that $G$ is quite close to being a line graph.

Let $\left(H, A_{1}, A_{2}, B_{1}, B_{2}\right)$ be an irreducible canonical interval 2-join in $G$. Note that $G$ has no simplicial vertices, since $\delta(G) \geq \Delta(G)-1$ and $\omega(G)<\Delta(G)$. Label the vertices of $H$ left-to-right as $v_{1}, \ldots, v_{t}$. Say $A_{1}=\left\{v_{1}, \ldots, v_{L}\right\}$ and $A_{2}=\left\{v_{R}, \ldots, v_{t}\right\}$. For $v \in V(H)$, let $r(v):=\max \left\{i \in[t] \mid v \leftrightarrow v_{i}\right\}$ and $l(v):=\min \left\{i \in[t] \mid v \leftrightarrow v_{i}\right\}$. These are well-defined since $|H| \geq 2$ and $H$ is connected by the following claim.

Claim 0. $H$ is connected and each of $A_{1}, A_{2}, B_{1}, B_{2}$ is nonempty. Otherwise $G$ contains a simplicial vertex.

Claim 1. If $H$ is incomplete, then $r\left(v_{L}\right)=r\left(v_{1}\right)+1$ and $l\left(v_{R}\right)=l\left(v_{t}\right)-1$. In particular, $v_{1}$ and $v_{t}$ are low and also $\left|A_{1}\right| \geq 2$ and $\left|A_{2}\right| \geq 2$. Suppose instead that $H$ is incomplete and $r\left(v_{L}\right) \neq r\left(v_{1}\right)+1$. By definition, $N_{H}\left(v_{1}\right) \subseteq N_{H}\left(v_{L}\right)$ and $v_{1}$ and $v_{L}$ have the same neighbors in $G \backslash H$. If $r\left(v_{L}\right)=r\left(v_{1}\right)$, then $N_{H}\left(A_{1}\right) \backslash A_{1}=N_{H}\left(v_{1}\right) \backslash A_{1}$, so $H$ is reducible, which is 
a contradiction. Thus $r\left(v_{L}\right) \geq r\left(v_{1}\right)+1$. If $r\left(v_{L}\right) \geq r\left(v_{1}\right)+2$, then $d\left(v_{L}\right)-d\left(v_{1}\right) \geq 2$, which is impossible, since $\delta(G) \geq \Delta(G)-1$. So $r\left(v_{L}\right)=r\left(v_{1}\right)+1$, as desired. Similarly, $l\left(v_{R}\right)=l\left(v_{t}\right)-1$.

Claim 2. If $H$ is complete or complete less an edge, then $R-L=1$. Assume, for a contradiction, that $R-L \neq 1$, so $V(H) \neq A_{1} \cup A_{2}$. First suppose that $H$ is complete. Now any $v \in V(H) \backslash A_{1} \cup A_{2}$ is simplicial in $G$, which is a contradiction. So suppose instead that $H$ is complete less an edge, and choose $v \in V(H) \backslash\left(A_{1} \cup A_{2}\right)$. Now $N[v]$ is complete less an edge; since $G$ has no $K_{\Delta}, v$ must be low. By Claim $1, v_{1}$ and $v_{t}$ are also low, so $G$ contains a copy of $K_{4}-e$ in which one vertex in both triangles is high and the other three vertices are low. This subgraph is $f$-AT, as shown in Figure $1 \mathbb{1}($ a), which is a contradiction.

Claim 3. $B_{1} \nsubseteq B_{2}, B_{2} \nsubseteq B_{1}$. If not, then by symmetry we can assume $B_{2} \subseteq B_{1}$. First, suppose $H$ is complete or complete less an edge. By Claim 2, $R-L=1$. If $H$ is complete, then the vertices in $A_{2}$ are simplicial, which is impossible. If $H$ is complete less an edge, then for a high vertex $v$ in $A_{2}$ (which exists by Claim 1), $N[v]$ induces $K_{\Delta+1}-e$; this contains $K_{\Delta}$, which is a contradiction.

So $H$ is neither complete nor complete less an edge; in particular $v_{1} \leftrightarrow v_{t}$. If $v_{1} \leftrightarrow v_{t-1}$, then $v_{t-1}$ is high, since $d\left(v_{t}\right)<d\left(v_{t-1}\right)$. This implies $v_{t} \leftrightarrow v_{2}$; now $H$ is complete less an edge, which is a contradiction. So $v_{1} \nLeftarrow v_{t-1}$ and, by symmetry, $v_{2} \leftrightarrow v_{t}$. If $\left|B_{2}\right| \geq 2$, then since $\left|A_{1}\right| \geq 2$ and $\left|A_{2}\right| \geq 2$ by Claim 1 , then consider the subgraph induced by $v_{1}, v_{L}, V_{R}, v_{t}$, and two vertices of $B_{2}$. Since $B_{2} \subseteq B_{1}$, this induced subgraph is either Figure 4(a) or Figure 4(b), which is a contradiction, since $G$ is $B K$-free.

So we must have $\left|B_{2}\right|=1$. Let $\{w\}=B_{2}$. Now $v_{t}$ is in a $K_{\Delta-1}$ in $H$, say with vertices $v_{q}, v_{q+1}, \ldots, v_{t}$. In particular, $w$ is not joined to $H$, so $R-L \neq 1$. If $\left|A_{2}\right| \geq 4$, then $\left\{v_{t}, v_{t-1}, v_{t-2}, v_{t-3}, v_{q}, w\right\}$ induces $K_{4} \vee E_{2}$, where the $K_{4}$ has a low vertex, $v_{t}$. As shown in Figure [i](d), this is $f$-AT, which is a contradiction. So $\left|A_{2}\right| \leq 3$.

First, suppose $v_{R-1}$ is low. Now $l\left(v_{R-1}\right)=q-1$. Since $\left|A_{2}\right| \leq 3$, the subgraph induced by $\left\{v_{t}, v_{R-1}, v_{R-2}, v_{R-3}, v_{R-4}, v_{q-1}\right\}$ is $K_{4} \vee E_{2}$, with a low vertex in the $E_{2}$. This is $f$-AT by Figure[1](d), which is a contradiction. So assume instead that $v_{R}$ is high. Now $l\left(v_{R-1}\right)=q-2$, so the subgraph induced by $\left\{v_{t}, v_{t-1}, v_{R-1}, v_{R-2}, v_{R-3}, v_{R-4}, v_{q-1}, v_{q-2}\right\}$ is $K_{4} \vee B$, where $B$ is not almost complete. This subgraph is $f$-AT, as shown in Figures [1(g) [I] (i), which is a contradiction.

Claim 4. $\left|A_{1}\right|,\left|A_{2}\right| \leq 3$. Suppose otherwise, by symmetry, that $\left|A_{1}\right| \geq 4$. First, suppose $H$ is complete. By Claim 2, $V(H)=A_{1} \cup A_{2}$. If $v_{1}$ is low, then for any $w_{1} \in B_{1} \backslash B_{2}$ the vertex set $\left\{v_{1}, \ldots, v_{4}, v_{t}, w_{1}\right\}$ induces a $K_{4} \vee E_{2}$, which contradicts Figures [][(d), Hence $v_{1}$ is high. If $\left|A_{2}\right| \geq 2$ and $\left|B_{1} \backslash B_{2}\right| \geq 2$, then for any $w_{1}, w_{2} \in B_{1} \backslash B_{2}$, the vertex set $\left\{v_{1}, \ldots, v_{4}, v_{t-1}, v_{t}, w_{1}, w_{2}\right\}$ induces $K_{4} \vee 2 K_{2}$, which contradicts Figure 1](g). Hence either $\left|A_{2}\right|=1$ or $\left|B_{1} \backslash B_{2}\right|=1$. Suppose $\left|A_{2}\right|=1$. Since $A_{1} \cup B_{1}$ induces a clique and $\left|A_{1} \cup B_{1}\right|=$ $d\left(v_{1}\right), v_{1}$ must be low, which is impossible. Hence, we have $\left|B_{1} \backslash B_{2}\right|=1$, so $\left|B_{1} \cap B_{2}\right|=$ $\left|B_{1}\right|-1$. Hence, $V(H) \cup\left(B_{1} \cap B_{2}\right)$ induces a clique of size $\left|A_{1}\right|+\left|A_{2}\right|+\left|B_{1}\right|-1=d\left(v_{1}\right)=\Delta$, which is a contradiction.

So $H$ must be incomplete. By Claim $1, v_{1}$ is low. Now, as above, for any $w_{1} \in B_{1} \backslash B_{2}$, the vertex set $\left\{v_{1}, \ldots, v_{4}, v_{L+1}, w_{1}\right\}$ induces a $K_{4} \vee E_{2}$ that contradicts Figure $\mathbb{1}(\mathrm{d})$ ). Hence, $\left|A_{1}\right| \leq 3$ and, by symmetry, $\left|A_{2}\right| \leq 3$.

Claim 5. $R-L=1$. Suppose otherwise that $R-L \geq 2$. By Claim 2, $H$ is incomplete. Now by Claim 1, $r\left(v_{L}\right)=r\left(v_{1}\right)+1, l\left(v_{R}\right)=l\left(v_{t}\right)-1, v_{1}$ and $v_{t}$ are low, and $\left|A_{1}\right| \geq 2$ and 
$\left|A_{2}\right| \geq 2$. Now we will find an $f$-AT subgraph induced by some vertices of $H$. To this end, we describe $N\left(v_{L+1}\right), N\left(v_{L+2}\right), N\left(v_{L+3}\right), N\left(v_{L+4}\right)$.

Subclaim 5a. $L+\Delta-2 \leq r\left(v_{L+1}\right) \leq L+\Delta-1$. Since $v_{L+1}$ has exactly $L$ neighbors to the left, $r\left(v_{L+1}\right) \leq L+1+\Delta-L=\Delta+1 \leq L+\Delta-1$. If $v_{L+1}$ is high, this computation is exact, so $r\left(v_{L+1}\right)=\Delta+1 \geq L+\Delta-2$. So suppose instead that $v_{L+1}$ is low. If $L=3$, then for some $w_{1} \in B_{1}$ the vertex set $\left\{v_{1}, v_{2}, v_{3}, v_{4}, w_{1}\right\}$ induces a $K_{3} \vee E_{2}$ that contradicts Figure1(b). Hence $L=2$ and $r\left(v_{L+1}\right)=L+1+\Delta-1-L=\Delta \geq L+\Delta-2$.

Subclaim 5b. $L+\Delta-2 \leq r\left(v_{L+2}\right) \leq L+\Delta$. By Subclaim 5a, $r\left(v_{L+2}\right) \geq L+\Delta-2$. Since $H$ contains no $\Delta$-clique, $v_{L+2}$ has at least 2 neighbors to the left if it is high and at least 1 neighbor to the left if it is low. Thus $r\left(v_{L+2}\right) \leq L+2+\Delta-2=L+\Delta$.

Subclaim 5c. If $v_{L+4}$ is high, then $l\left(v_{L+4}\right) \leq L$. Suppose otherwise. Recall that $v_{L+1} \leftrightarrow$ $v_{L+4}$, since $d\left(v_{L+1}\right) \geq \Delta-1 \geq 8$ and $\left|A_{1}\right| \leq 3$. Now $v_{L+4}$ has exactly 3 neighbors to the left, so $r\left(v_{L+4}\right)=L+\Delta+1$. Consider the subgraph induced on $\left\{v_{L+1}, v_{L+2}, v_{L+4}, v_{L+5}, v_{L+6}, v_{L+7}\right.$, $\left.v_{L+9}, v_{L+10}\right\}$. By Subclaims 5a and 5b, this subgraph contradicts Figure 1] $[(\mathrm{g})]$ or Figure [1](h)]

Subclaim 5d. $l\left(v_{L+3}\right) \leq L$. Suppose otherwise. Since $v_{L+1} \leftrightarrow v_{L+3}$, vertex $v_{L+3}$ has exactly 2 neighbors to the left, so $r\left(v_{L+3}\right) \geq L+\Delta$. By Subclaim $5 \mathrm{c}, v_{L+4}$ is low. By Subclaim 5a, $L+\Delta-2 \leq r\left(v_{L+1}\right) \leq L+\Delta-1$. Therefore $\left\{v_{L+1}, v_{L+3}, v_{L+4}, v_{L+5}, v_{L+6}, v_{L+\Delta}\right\}$ induces a $K_{4} \vee E_{2}$ that contradicts Figure [][d $)$.

Subclaim 5e. $r\left(v_{1}\right) \geq L+2$. By Subclaim $5 \mathrm{~d}, r\left(v_{L}\right) \geq L+3$, so Claim 1 implies that $r\left(v_{1}\right) \geq L+2$.

Subclaim 5f. Claim 5 is true. If $r\left(v_{r\left(v_{1}\right)-1}\right)=r\left(v_{1}\right)+1$, then $v_{r\left(v_{1}\right)-1}$ is low, so $\left\{v_{1}, v_{L+1}, v_{L+2}, v_{L+3}, v_{r\left(v_{1}\right)-1}, v_{r\left(v_{1}\right)+1}\right\}$ induces $K_{4} \vee E_{2}$, which contradicts Figure [1](d). So assume $r\left(v_{r\left(v_{1}\right)-1}\right) \neq r\left(v_{1}\right)+1$.

Consider the subgraph $Q$ induced on $\left\{v_{1}, v_{L}, v_{r\left(v_{1}\right)-1}, v_{r\left(v_{1}\right)}, v_{r\left(v_{1}\right)+1}, v_{r\left(v_{r\left(v_{1}\right)-1}\right)}\right\}$; these vertices must be distinct. Both $v_{r\left(v_{1}\right)-1}$ and $v_{r\left(v_{1}\right)}$ are dominating vertices in $Q$. We show that $\left\{v_{1}, v_{L}, v_{r\left(v_{1}\right)+1}, v_{r\left(v_{r\left(v_{1}\right)-1}\right)}\right\}$ induces a $P_{4}$, so $Q$ is Figure [i[ (b)], which is a contradiction. By definition, $v_{1} \leftrightarrow v_{L}, v_{1} \leftrightarrow v_{r\left(v_{1}\right)+1}$, and $v_{1} \leftrightarrow v_{r\left(v_{r\left(v_{1}\right)-1}\right)}$. By Claim 2, $v_{L} \leftrightarrow v_{r\left(v_{1}\right)+1}$. By Subclaim 5e, $r\left(v_{1}\right) \geq L+2$, so $r\left(v_{1}\right)-1 \geq L+1$. Since $\left|B_{1}\right|>0$ by Claim 0 , this means $r\left(v_{r\left(v_{1}\right)-1}\right)-\left(r\left(v_{1}\right)-1\right) \geq r\left(v_{L}\right)-L$ and hence $r\left(v_{r\left(v_{1}\right)-1}\right) \geq r\left(v_{L}\right)-L+\left(r\left(v_{1}\right)-1\right) \geq r\left(v_{L}\right)+1$. Therefore $v_{L} \nLeftarrow v_{r\left(v_{r\left(v_{1}\right)-1}\right)}$, so $\left\{v_{1}, v_{L}, v_{r\left(v_{1}\right)+1}, v_{r\left(v_{r\left(v_{1}\right)-1}\right)}\right\}$ induces a $P_{4}$ as desired.

Claim 6. $B_{1} \cap B_{2}=\emptyset$. Suppose otherwise that we have $w \in B_{1} \cap B_{2}$.

Subclaim 6a. Each $v \in V(H)$ is low, $\left|B_{1}\right|=\left|B_{2}\right|,\left|B_{1} \backslash B_{2}\right|=\left|B_{2} \backslash B_{1}\right|=1, d(v)=$ $\left|A_{1}\right|+\left|A_{2}\right|+\left|B_{1}\right|-1$ for each $v \in V(H)$ and $H$ is complete. By Claim 5, we have $d(v) \leq\left|A_{1}\right|+\left|A_{2}\right|+\left|B_{1}\right|-1$ for each $v \in A_{1}$ and $d(v) \leq\left|A_{1}\right|+\left|A_{2}\right|+\left|B_{2}\right|-1$ for each $v \in A_{2}$. Since $B_{1} \nsubseteq B_{2}$ and $B_{2} \nsubseteq B_{1}$, we have $d(w) \geq \max \left\{\left|B_{1}\right|,\left|B_{2}\right|\right\}+\left|A_{1}\right|+\left|A_{2}\right|$. So $d(w) \geq d(v)+1$ for every $v \in V(H)$. This implies that each $v \in V(H)$ is low, $\left|B_{1}\right|=\left|B_{2}\right|$, $\left|B_{1} \backslash B_{2}\right|=\left|B_{2} \backslash B_{1}\right|=1$, and $d(v)=\left|A_{1}\right|+\left|A_{2}\right|+\left|B_{1}\right|-1$ for each $v \in V(H)$. Hence, $H$ is complete.

Subclaim 6b. $\left|B_{1} \cap B_{2}\right| \leq 3$. Suppose otherwise that $\left|B_{1} \cap B_{2}\right| \geq 4$. Pick $w_{1} \in$ $B_{1} \backslash B_{2}, w_{2} \in B_{2} \backslash B_{1}$ and $z_{1}, z_{2}, z_{3}, z_{4} \in B_{1} \cap B_{2}$. Since $v_{1} \nLeftarrow w_{2}$ and $v_{t} \not s w_{1}$, the set $\left\{z_{1}, z_{2}, z_{3}, z_{4}, w_{1}, w_{2}, v_{1}, v_{t}\right\}$ induces an $f$-AT subgraph shown in Figure [1](g), [1](h), or Figure [1(i); each case yields a contradiction. Hence $\left|B_{1} \cap B_{2}\right| \leq 3$.

Subclaim 6c. Claim 6 is true. By Subclaim 6a and Subclaim $6 \mathrm{~b}$ we have $3 \geq$ $\left|B_{1} \cap B_{2}\right|=\left|B_{1}\right|-1$, so $\left|B_{1}\right|=\left|B_{2}\right| \leq 4$. If $\left|A_{1}\right| \leq 2$ and $\left|A_{2}\right| \leq 2$, then $\Delta-1=d\left(v_{1}\right) \leq$ 
$3+\left|B_{1}\right| \leq 7$, which is a contradiction. Hence, by symmetry, we assume that $\left|A_{1}\right| \geq 3$. But now for any $w_{1} \in B_{1} \backslash B_{2}$, the set $\left\{v_{1}, v_{2}, v_{3}, v_{t}, w_{1}\right\}$ induces a $K_{3} \vee E_{2}$ contradicting Figure [1](b).

Claim 7. Either $H$ is complete, or $H=K_{|H|}-x y$ where $x$ and $y$ are low in $G$ and $|H| \leq 6$. Suppose $H$ is incomplete. By Claim $5, R-L=1$. So, by Claim $1 r\left(v_{L}\right)=r\left(v_{1}\right)+1$ and $l\left(v_{R}\right)=l\left(v_{t}\right)-1$. Since $v_{1}$ is not simplicial, $r\left(v_{1}\right) \geq L+1=R$. Hence $l\left(v_{R}\right)=1$, so $l\left(v_{t}\right)=2$. Similarly, $r\left(v_{1}\right)=t-1$. So, $H$ is $K_{t}$ less an edge and $v_{1}$ and $v_{t}$ are low (by Claim 2). Finally, by Claims 5 and $4,|H|=\left|A_{1}\right|+\left|A_{2}\right| \leq 3+3=6$.

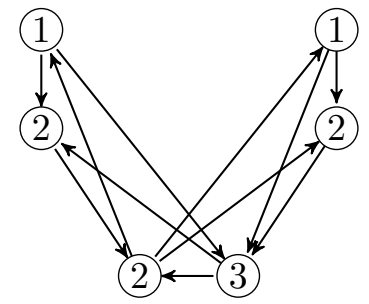

(a) $\mathrm{EE}=8, \mathrm{EO}=9$

$K_{2} \vee 2 K_{2}$ with two lows

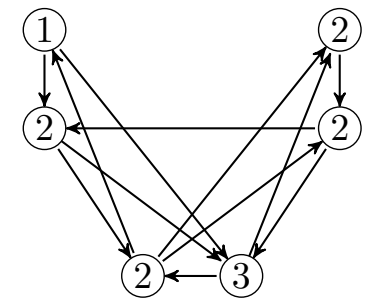

(b) $\mathrm{EE}=14, \mathrm{EO}=15$

$K_{2} \vee P_{4}$ with one low end

Figure 4: Subgraphs forbidden by Alon-Tarsi orientations, used in Lemma 2.3.

Recall our goal in this section: to write $G$ as a composition of linear interval strips, where each strip is complete or complete less an edge (since this implies that $G$ is very nearly a line graph). Our main tool in this endeavor is Lemma 2.3, which we will apply to each interval 2 -join in the representation. To this end, we would like that every interval 2 -join is canonical and irreducible. Of course, trivial 2-joins are fine also, since their strips must be complete. So in Lemma 2.4, we show that every interval 2-join is either trivial or canonical. Finally, in the proof of Lemma 2.5 we choose a composition representation with the maximum number of strips; thus, every canonical interval 2-join is irreducible.

Lemma 2.4. Let $G$ be a BK-free graph with $\Delta(G) \geq 9$ and $\omega(G)<\Delta(G)$. Each interval 2-join in $G$ is either trivial $\left(A_{1}=A_{2}\right)$ or canonical $\left(A_{1} \cap A_{2}=\emptyset\right)$.

Proof. Let $\left(H, A_{1}, A_{2}, B_{1}, B_{2}\right)$ be an interval 2-join in $G$. Suppose that $H$ is nontrivial $\left(A_{1} \neq A_{2}\right)$ and let $C:=A_{1} \cap A_{2}$. Now $\left(H \backslash C, A_{1} \backslash C, A_{2} \backslash C, C \cup B_{1}, C \cup B_{2}\right)$ is a canonical interval 2-join. We reduce this 2-join until we get an irreducible canonical interval 2-join $\left(H^{\prime}, A_{1}^{\prime}, A_{2}^{\prime}, B_{1}^{\prime}, B_{2}^{\prime}\right)$ with $H^{\prime} \unlhd H \backslash C$. Since $C$ is joined to $H \backslash C$, it is also joined to $H^{\prime}$. Hence $C \subseteq B_{1}^{\prime} \cap B_{2}^{\prime}$. Now Lemma 2.3 implies $B_{1}^{\prime} \cap B_{2}^{\prime}=\emptyset$, so $A_{1} \cap A_{2}=C=\emptyset$. Thus, $H$ is canonical.

Lemma 2.5. If $G$ is a quasi-line BK-free graph with $\Delta(G) \geq 9$ and $\omega(G)<\Delta(G)$, then there is a line graph $G^{\prime}$ with $G \subseteq G^{\prime}$ such that $\Delta\left(G^{\prime}\right)=\Delta(G)$ and $\omega\left(G^{\prime}\right)<\Delta\left(G^{\prime}\right)$.

Proof. By Lemma 2.1, $G$ is not a circular interval graph. By Lemma 2.2, $G$ has no non-linear homogeneous pair of cliques. So, by Theorem [1.3. $G$ is a composition of linear interval strips. Choose such a composition representation of $G$ using the maximum number of strips. By Lemma 2.4, every interval 2-join is trivial or canonical. 
Let $\left(H, A_{1}, A_{2}\right)$ be a strip in the composition. Let $B_{1}:=N_{G \backslash H}\left(A_{1}\right)$ and $B_{2}:=N_{G \backslash H}\left(A_{2}\right)$. Now $\left(H, A_{1}, A_{2}, B_{1}, B_{2}\right)$ is an interval 2-join. If $A_{1}=A_{2}$, then $H$ is complete. So suppose $A_{1} \neq A_{2}$. Now $H$ is canonical, by Lemma 2.4. If $H$ is reducible, then by symmetry we can assume that $N_{H}\left(A_{1}\right) \backslash A_{1}=N_{H}\left(v_{1}\right) \backslash A_{1}$. But now replacing the strip $\left(H, A_{1}, A_{2}\right)$ with the two strips $\left(G\left[A_{1}\right], A_{1}, A_{1}\right)$ and $\left(H \backslash A_{1}, N_{H}\left(A_{1}\right) \backslash A_{1}, A_{2}\right)$ gives a composition representation of $G$ using more strips, which is a contradiction. Hence $H$ is irreducible. Now by Lemma 2.3, $H$ is complete or $K_{t}-x y$ where $x$ and $y$ are low in $G$ and $t \leq 6$. Thus, $G$ is a composition of strips, each of which is either complete or $K_{t}-x y$, where $x$ and $y$ are low in $G$ and $t \leq 6$.

Note that each vertex can play the role of $x$ or $y$ in at most one incomplete strip. So, we can add a matching containing $x y$ for each strip of the form $K_{t}-x y$ without increasing the maximum degree. Let $G^{\prime}$ be the resulting graph. Now we show that adding this matching does not create a $K_{\Delta(G)}$. For each strip of the form $K_{t}-x y$, exactly one maximal clique in $G^{\prime}$ contains both $x$ and $y$ (since $B_{1} \cap B_{2}=\emptyset$ ) and this clique has at most 6 vertices. Hence $\omega\left(G^{\prime}\right) \leq \max \{6, \omega(G)\}<\Delta(G)$, as desired.

Lemma 2.5 completes our proof that if the list-coloring version of the Borodin-Kostochka conjecture (or its paintability analogue) is true for line graphs, then it is true for quasi-line graphs. If $G$ is a quasi-line counterexample, then the $G^{\prime}$ guaranteed by the lemma is a line-graph counterexample, since $\chi_{\ell}\left(G^{\prime}\right) \geq \chi_{\ell}(G)$; similarly, $\chi_{O L}\left(G^{\prime}\right) \geq \chi_{O L}(G)$.

\section{Line Graphs}

In this section we consider line graphs. The general idea is to show that if $G$ is a line graph of $H$, then some subgraph of $G$ is a line graph of a bipartite graph $B$ such that each edge of $B$ has many of its adjacent edges also in $B$. We then use a result of Borodin, Kostochka, and Woodall [6] to show that the line graph of $B$ is $f$-AT or $f$-KP. This is the first place in our proof that relies heavily on subgraphs being kernel-perfect. In particular, the key result from [6], shown below as Theorem [3.2, has no analogue for Alon-Tarsi orientations. (One example of this is that the line graph of $K_{3,3}$ has no Alon-Tarsi orientation with maximum outdegree at most 2.) Our main result in this section is the following theorem.

Theorem 3.1. If $G$ is a BK-free line graph, then $\Delta(G)<69$. Thus, if $G$ is a line graph with $\Delta(G) \geq 69$, then $\chi_{O L}(G) \leq \max \{\omega(G), \Delta(G)-1\}$.

In other words, we prove the Borodin-Kostochka conjecture, strengthened to online listcoloring, for the class of line graphs with maximum degree at least 69. (When combined with the previous section, this proves the same result for the larger class of quasi-line graphs with maximum degree at least 69.) Our proof relies mainly on the kernel method. This technique came to prominence when Galvin [14] used it to prove the List Coloring Conjecture for line graphs of bipartite graphs. More precisely, he showed that if $G$ is the line graph of a bipartite graph $H$, then $G$ is $\Delta(H)$-edge-choosable. A few years later Borodin, Kostochka, and Woodall [6] sharpened Galvin's result. They proved the following. (They only stated the result for list-coloring, but the same proof gives the result for kernel-perfection.)

Theorem 3.2 ([6]). If $G$ is the line graph of a bipartite graph $B$, then $G$ is $f-K P$, where $f(u v)=\max \left\{d_{B}(u), d_{B}(v)\right\}$ for every edge uv in B. Thus, $G$ is $f$-paintable. 
This strengthening allowed for a surprisingly wide range of applications. One beautiful consequence of Theorem 3.2 is that for every constant $k$ there exists a constant $\Delta_{k}$ such that if $\operatorname{mad}(G)<k$ and $\Delta(G) \geq \Delta_{k}$, then $\chi_{\ell}^{\prime}(G)=\chi^{\prime}(G)$. The main idea of the proof is to find as a subgraph of $G$ a certain type of bipartite graph $B$ such that any coloring of $E(G) \backslash E(B)$ can be extended to $E(B)$ by Theorem 3.2. Recently, Woodall [24] gave a simpler proof of this result. In that paper he made explicit that it suffices to let $\Delta_{k}=\frac{k^{2}}{2}$. Since all of these proofs use the kernel method, they extend directly to online list-coloring, as observed by Schauz [23].

Galvin's proof is well-known and it has been widely reproduced (for example, in [1] and [13]). The proofs for the extensions by Borodin, Kostochka, and Woodall [6] and Schauz [23] are similar, so we do not reproduce them here. However, the result for bounded maximum average degree is much less well-known. (Further, we need one extra wrinkle, since the proofs in [6] and 24] give an upper bound on $\Delta(H)$. We must translate this to an upper bound on $\Delta(G)$, but this final step is relatively easy.) We particularly like Woodall's presentation, so we follow that below, in Theorem 3.8 .

The proof of our main result in this section has a simple outline. Let $G$ be the line graph of some graph $H$. In Lemmas 3.3 3.6, we show that if $G$ is BK-free, then $H$ is 6-degenerate. In particular, $\operatorname{mad}(H)<12$. Next, in Lemma 3.7 and Theorem 3.8 , we apply Theorem 3.2 to show that if $G$ is BK-free and has $\operatorname{mad}(H)<12$, then $\Delta(G) \leq 68$. This completes the proof for line graphs.

Now we recall how this section fits into the larger context of the paper. In the previous section, we showed that if there exists a BK-free quasi-line graph $G$ with $\Delta(G) \geq 9, \omega(G)<$ $\Delta(G)$, and $\chi(G)>\max \{\omega(G), \Delta(G)-1\}$, then there exists such a $G$ that is a line graph. In fact, the proof constructs the line graph with the same maximum degree as the original. Thus, our result that $\chi_{O L}(G) \leq \max \{\omega(G), \Delta(G)-1\}$ for every line graph $G$ with $\Delta(G) \geq 69$ immediately extends to prove the same bound for every quasi-line graph $G$ with $\Delta(G) \geq 69$. Combining this result with our previous work (Theorem 5.6 in [10]), we get that $\chi_{\ell}(G) \leq$ $\max \{\omega(G), \Delta(G)-1\}$ for every claw-free graph $G$ with $\Delta(G) \geq 69$.

In the rest of this section, we prove Theorem 3.1. We begin with two lemmas showing that certain graphs are $d_{1}$-AT or $d_{1}$-KP. The hypothesis $\omega(G)<\Delta(G)$ arises naturally from our interest in the Borodin-Kostochka Conjecture. When $G$ is a line graph of $H$, the edges incident to any common endpoint in $H$ form a clique in $G$, so $\Delta(H) \leq \omega(G)<\Delta(G)$.

Lemma 3.3. If $G$ is BK-free with $\omega(G)<\Delta(G)$ and $G$ is the line graph of some graph $H$, then $\mu(H) \leq 3$. Further, no edge of multiplicity 3 in $H$ appears on a triangle.

Proof. Suppose, to the contrary, that $H$ has some edge $e$ of multiplicity at least 4; let $v \in V(G)$ be a vertex corresponding to $e$. First suppose that $d_{G}(v)=\Delta(G)$. Now $G[\{v\} \cup$ $N(v)]=K_{4} \vee B$, for some graph $B$, since $e$ has multiplicity at least 4 . Since $\omega(G)<\Delta(G)$, we get that $\omega(B) \leq|B|-2$. Since $G$ is a line graph, $B$ has independence number 2, so $B$ contains two disjoint pairs of non-adjacent vertices. Thus, $K_{4} \vee B$ is $d_{1}$-AT, as shown in Figure [1] (g) (i). Now suppose instead that $d_{G}(v)=\Delta(G)-1$. The argument is essentially the same; however, now we only get that $\omega(B) \leq|B|-1$, so $B$ is incomplete. Now $K_{4} \vee B$ is $f$-AT, as shown in Figure $1(\mathrm{~d})$, since $v$ is low. This completes the proof of the first statement.

Now we prove the second statement. Suppose, to the contrary, that $H$ has an edge $e$ of multiplicity 3 on a triangle. Let $x_{1}, x_{2}, x_{3}$ be the vertices of the triangle, with $x_{1}$ and $x_{2}$ 
the endpoints of $e$. Let $v_{1}, v_{2}, v_{3}$ be the vertices corresponding to edges with endpoints $x_{1}$ and $x_{2}$. Let $v_{4}$ and $v_{5}$ be vertices corresponding to edges $x_{1} x_{3}$ and $x_{2} x_{3}$. Similar to above, $G[\{v\} \cup N(v)]=K_{3} \vee B$, for some graph $B$. If $d_{G}(v)=\Delta(G)-1$, then $\omega(G) \leq|B|-1$. So some edge of $H$ incident to $x_{1}$ or $x_{2}$ has an endpoint outside of $\left\{x_{1}, x_{2}, x_{3}\right\}$. By symmetry, say it is incident $x_{1}$; let $v_{6}$ be the corresponding vertex of $G$. Now $v_{1}, \ldots, v_{6}$ induce in $G$ a subgraph that is $f$-AT, as shown in Figure 1 $1(\mathrm{~d})$, since $v_{1}$ is low.

Assume instead that $d_{G}(v)=\Delta(G)$, so $\omega(G) \leq|B|-2$. Recall that $K_{3} \vee P_{4}$ is $d_{1^{-}}$ AT, as shown in Figure [1/(h). Suppose that an edge incident to $x_{1}$ has an endpoint outside $\left\{x_{1}, x_{2}, x_{3}\right\}$ and also that an edge incident to $x_{2}$ has an endpoint outside $\left\{x_{1}, x_{2}, x_{3}\right\}$. If these endpoints are distinct, then $G$ has a copy of $K_{3} \vee P_{4}$, which is $d_{1}$-AT. If these endpoints are identical, then $G$ has a copy of $K_{2} \vee C_{4}$, which is $d_{1}$-AT, as shown in Figure [1](i). So we conclude that either $x_{1}$ or $x_{2}$ has no incident edges with endpoints outside of $\left\{x_{1}, x_{2}, x_{3}\right\}$; by symmetry, assume it is $x_{2}$. Now we can view $K_{3} \vee B$ as $K_{4} \vee\left(B-v_{4}\right)$, since $v_{4}$ dominates $B$. Since $\omega(B) \leq|B|-2$, we conclude that $B-v_{4}$ contains two disjoint pairs of nonadjacent vertices. Thus, $K_{4} \vee\left(B-v_{4}\right)$ is $d_{1}$-AT, as shown in Figure [1] $(\mathrm{g})$.

Before proving our next lemma, we need a bit more information about kernel-perfect orientations. We can easily show that if $D$ is a kernel-perfect digraph, then every clique of $D$ is oriented transitively (possibly with some bidirected edges); otherwise $D$ would have some cyclically oriented 3-cycle, which has no kernel. Further, every directed odd cycle must have a chord. In general, these condition are not sufficient to imply that $D$ is kernel-perfect. However Borodin, Kostochka, and Woodall [7] showed that if the underlying undirected graph $G$ of $D$ is a line graph, then these conditions are indeed sufficient.

Theorem $3.4([7])$. Let $H$ be a line graph of a multigraph. An orientation $D$ of $H$ is kernelperfect if and only if every clique of $H$ is transitively oriented (possibly with some bidirected edges) and every directed odd cycle of D has a chord (also possibly bidirected).

Now we use Theorem 3.4 to prove that three particular line graphs have $f$-KP orientations, where $f(v)=d(v)$ for a few specified vertices $v$ and $f(v)=d(v)-1$ otherwise.

Lemma 3.5. The line graphs of the subgraphs shown in Figure 5 are $f$-KP, where $f(v)=$ $d(v)$ for vertices corresponding to the six bold edges in (b) and $f(v)=d(v)-1$ otherwise.

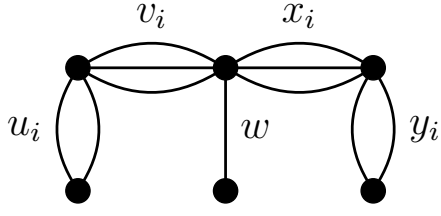

(a)

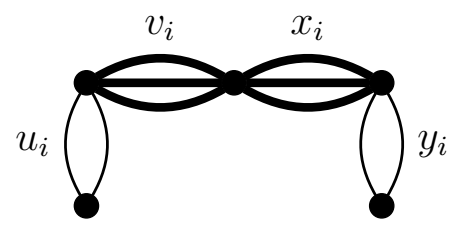

(b)

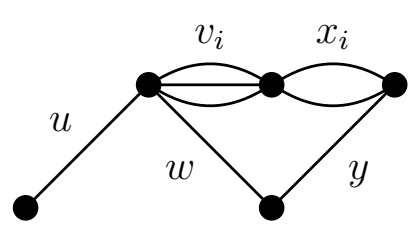

(c)

Figure 5: The three cases of Lemma 3.5.

Proof. In each case, let $H$ denote the graph shown and let $G$ denote its line graph. The orientation for the second line graph comes from the orientation for the first, simply by 
deleting a vertex. Since the lists sizes don't go down, the reducibility of the first line graph implies the reducibility of the second. Our orientations $D$ will actually orient some edges in both directions. This is fine, as long as $d_{D}^{+}(v) \leq d_{G}(v)-2$.

We begin with Figure 5(a). We refer to the edges of the subgraph and the vertices of its line graph interchangably. From left to write, label the edges as $u_{1}, u_{2}, v_{1}, v_{2}, v_{3}, w, x_{1}, x_{2}$, $x_{3}, y_{1}, y_{2}$; if vertices differ only in their subscript, then they correspond to parallel edges.

To form $D$, take all of the directed edges implied by transitivity in the three linear orders $v_{1} \rightarrow v_{2} \rightarrow u_{1} \rightarrow u_{2} \rightarrow v_{3} ; x_{1} \rightarrow x_{2} \rightarrow y_{1} \rightarrow y_{2} \rightarrow x_{3} ; v_{3} \rightarrow x_{3} \rightarrow w \rightarrow v_{1} \rightarrow v_{2} \rightarrow x_{1} \rightarrow x_{2}$ (one order for each maximal clique in $G$ ). Theorem 3.4 immediately implies that $D$ is kernelperfect, since $G$ is chordal. All that remains is to verify that the outdegrees are small enough. In the table below we give the degree of each vertex in $G$ and its outdegree in $D$.

\begin{tabular}{c|ccccccccccc} 
& $u_{1}$ & $u_{2}$ & $v_{1}$ & $v_{2}$ & $v_{3}$ & $w$ & $x_{1}$ & $x_{2}$ & $x_{3}$ & $y_{1}$ & $y_{2}$ \\
\hline$d_{G}$ & 4 & 4 & 8 & 8 & 8 & 6 & 8 & 8 & 8 & 4 & 4 \\
$d_{D}^{+}$ & 2 & 1 & 6 & 5 & 6 & 4 & 4 & 3 & 5 & 2 & 1
\end{tabular}

Figure 6: Degrees in $G$ and outdegrees in $D$, for Figure 5(a).

This completes the proof for Figure 5(a), and also for Figure 5(b).

Now consider Figure 5(c). From left to write, label the edges as $u, v_{1}, v_{2}, v_{3}, w, x_{1}, x_{2}, y$. To form $D$, take all directed edges implied by the four linear orders $v_{3}, w, u, v_{1}, v_{2} ; v_{1}, v_{2}, x_{1}, x_{2}, v_{3}$; $x_{1}, x_{2}, y ; y, w$ (one order for each maximal clique in $G$ ). Again, Theorem 3.4 immediately implies that $D$ is kernel-perfect (now $G$ is no longer chordal, but every chordless cycle is even, which is sufficient). Again, we need only verify that the outdegrees are small enough. In the table below we give the degree of each vertex in $G$ and its outdegree in $D$.

\begin{tabular}{c|cccccccc} 
& $u$ & $v_{1}$ & $v_{2}$ & $v_{3}$ & $w$ & $x_{1}$ & $x_{2}$ & $y$ \\
\hline$d_{G}$ & 4 & 6 & 6 & 6 & 5 & 5 & 5 & 3 \\
$d_{D}^{+}$ & 2 & 4 & 3 & 4 & 3 & 3 & 2 & 1
\end{tabular}

Figure 7: Degrees in $G$ and outdegrees in $D$, for Figure 5 (c).

This completes the proof of the lemma.

If one or both pairs of parallel edges incident to leaves in Figure 5(a) ended instead at distinct leaves, then the resulting line graph would be unchanged; so it is again $f$-KP. In proving our next lemma, we use this observation implicitly.

Lemma 3.6. Let $G$ be the line graph of some graph $H$. If $\delta(H) \geq 7$ and $\mu(H) \leq 3$, then $G$ is not BK-free. Thus, if $G$ is BK-free and $\omega(G)<\Delta(G)$, then $H$ is 6-degenerate.

Proof. We begin by proving the first statement. Choose a partition $\{A, B\}$ of $V(H)$ to

(1) maximize $\|A, B\|$; and subject to that to

(2) minimize $\sum_{x y \in E(A, B)} \mu(x y)^{2}$ 
Here (2) is just giving preference to things like 3 single edges over one triple edge.

Let $Q$ be the bipartite graph with parts $A$ and $B$ and edges $E(A, B)$. Note that $d_{Q}(x) \geq$ $d_{H}(x) / 2$ for all $x \in V(Q)$ by (1); otherwise we could move $x$ to the other part and increase $\|A, B\|$. For each $x \in V(Q)$, let $\mu(x)$ be the maximum multiplicity of an edge in $Q$ incident to $x$. We apply Theorem 3.2 to show that the line graph of $Q$ is a $d_{1}-\mathrm{KP}$ subgraph of $G$ (or else $G$ contains some subgraph from Lemma 3.5 that is $d_{1}-\mathrm{KP}$ ).

The hypothesis for Theorem 3.2 requires that $\max \left\{d_{Q}(x), d_{Q}(y)\right\} \leq\left(d_{Q}(x)+d_{Q}(y)-\right.$ $2-(\mu(x y)-1))-1$ if edge $x y$ is high. So it suffices to show that $d_{Q}(x) \geq \mu(x y)+2$ and $d_{Q}(y) \geq \mu(x y)+2$. Similarly, if $x y$ is a low edge, then we need $d_{Q}(x) \geq \mu(x y)+1$ and $d_{Q}(y) \geq \mu(x y)+1$. Since $\mu(H) \leq 3$, and $d_{Q}(z) \geq d_{H}(z) / 2$ for all $z \in V(Q)$, it would suffice to have $\delta(H) \geq 9$. Thus, we may assume $\delta(H) \leq 8$. Since $\delta(Q) \geq 4$, we may apply Theorem 3.2 unless there is a vertex $x$ with $d_{Q}(x)=4$ incident to a high edge $x y$ with $\mu(x y)=3$. So suppose this is true. We have two cases: $d_{H}(x)=8$ and $d_{H}(x)=7$.

$d_{H}(x)=8$ : By $(2), x$ must be incident to two multiplicity 3 edges and two multiplicity 1 edges; otherwise we could move $x$ to the other part of the partition and contradict that the partition is extremal. But now we have a $d_{1}$-AT subgraph, by Lemma 3.5 .

$d_{H}(x)=7$ : We have $d_{H}(x)+d_{H}(y)-\mu(x y)-1=\Delta(G)$. Since $d_{H}(x)=7$, we get $d_{H}(y)=\Delta(G)-3$. Pick $w \in N_{H}(y)-x$. Now $\Delta(G) \geq d_{H}(y)+d_{H}(w)-\mu(y w)-1$, so $\mu(y w)+4 \geq d_{H}(w) \geq 7$. Thus $\mu(y w) \geq 3$. But now $y$ is incident to two multiplicity 3 edges, so we again have a $d_{1}$-AT subgraph, by Lemma 3.5.

This second statement of the lemma follows from the first one. Note that $\mu(H) \leq 3$, by Lemma 3.3. If $H$ has a subgraph $H^{\prime}$ with $\delta\left(H^{\prime}\right) \geq 7$, then we apply the first part of the lemma to $H^{\prime}$ and conclude that $G$ is not BK-free. Instead every subgraph $H^{\prime}$ of $H$ must have a vertex of degree at most 6 . Thus, by definition, $H$ is 6 -degenerate.

Lemma 3.7. Let $G$ be the line graph of some graph $H$ such that $\Delta(H)<\Delta(G)$. If $G$ is $B K$-free, then $H$ has no bipartite subgraph $B$ such that for every edge $x y \in E(B)$ the endpoint of smaller degree has all of its incident edges in $H$ also appearing in $B$. In other words, $H$ cannot contain a bipartite subgraph $B$ such that for each $x y \in E(B)$ either $(a)$ $d_{B}(y) \geq d_{B}(x)=d_{H}(x) ;$ or $(b) d_{B}(x) \geq d_{B}(y)=d_{H}(y)$.

Proof. Suppose, to the contrary, that we have graphs $G, H$, and $B$ as in the lemma. Let $D$ denote the line graph of $B$. We use Theorem 3.2 to show that $D$ is $f$-AT, where $f(v):=$ $d_{D}(v)-1+\Delta(G)-d_{G}(v)$ for all $v \in V(D)$. This contradicts the fact that $G$ is BK-free.

Consider a vertex $v$ of $D$ and let $x y$ denote the corresponding edge in $B$. To apply Theorem 3.2, we must show that $f(v)$ is sufficiently large; namely, we must show that $d_{D}(v)-1+\Delta(G)-d_{G}(v) \geq \max \left\{d_{B}(x), d_{B}(y)\right\}$. By symmetry, assume that $d_{B}(y) \geq$ $d_{B}(x)=d_{H}(x)$. Recall that $d_{D}(v)=d_{B}(x)+d_{B}(y)-\mu(x y)-1$. Now $f(v)=d_{D}(v)-1+$ $\Delta(G)-d_{G}(v)=\left(d_{B}(x)+d_{B}(y)-\mu(x y)-1\right)-1+\Delta(G)-\left(d_{H}(x)+d_{H}(y)-\mu(x y)-1\right)=$ $d_{B}(y)-1+\Delta(G)-d_{H}(y) \geq d_{B}(y)$, since $\Delta(H)<\Delta(G)$.

To simplify our presentation of the key lemma from [6] and [24], we state it only for the specific case needed for our application: $\operatorname{mad}(G)<12$. However, the proof extends easily to the more general case that $\operatorname{mad}(H)<C$, for some constant $C$.

Lemma 3.8. Suppose that $G$ is BK-free, $G$ is the line graph of a graph $H$, and $\operatorname{mad}(H)<12$. If $\omega(G)<\Delta(G)$, then $\Delta(G) \leq 68$. 
Proof. Suppose the lemma is false, and let $G$ be a counterexample. We use the discharging method to get a contradiction; since we know $\operatorname{mad}(H)<12$, we use discharging on the vertices of $H$. Give each vertex $v \in V(H)$ initial charge $\operatorname{ch}(v)=d_{H}(v)$. We have 10 successive rounds of discharging, rounds 2 through 11 . On round $i$, each vertex of degree at most $i$ receives charge 1 from some high degree neighbor. Thus, for each $v$ with $d(v) \leq 11$, vertex $v$ receives charge 1 on a total of $12-d(v)$ rounds. Hence, each such vertex receives total charge $12-d(v)$, and ends with final charge $d(v)+(12-d(v))=12$. We also must verify that no vertex gives away too much charge. Thus, each vertex finishes with charge at least 12 , which contradicts our assumption that $\operatorname{mad}(H)<12$. The details forthwith.

For each round of discharging, we use Lemma 3.7 repeatedly. For each $i$ with $2 \leq i \leq 11$, let $V_{i}$ be the vertices of $H$ of degree at most $i$. Let $B_{i}$ be the bipartite subgraph of $H$ containing $V_{i}$ and all edges incident to $V_{i}$. Since $G$ is BK-free, $\delta(G) \geq \Delta(G)-1$. Thus, each edge $u v$ in $H$ has $d(u)+d(v)-2 \geq \Delta(G)-1$, so $d(u)+d(v) \geq \Delta(G)+1$. Since $\Delta(H) \leq \omega(G)<\Delta(G)$, we have $d(u)+d(v) \geq \Delta(H)+2$ for each edge uv. In particular, $\delta(H) \geq 2$. This also implies that for each $i$ with $2 \leq i \leq 11$ the set $V_{i}$ is independent in $H$.

For every $u \in V_{i}$, we have $d_{B_{i}}(u)=d_{H}(u)$, so Lemma 3.7 shows that there must exist $v \in V\left(B_{i}\right) \backslash V_{i}$ with $d_{B_{i}}(v)<d_{B_{i}}(u) \leq i$. Thus, on round $i$, we give charge 1 from $v$ to each of its neighbors in $B_{i}$; afterwards, we delete from $B_{i}$ vertex $v$ and all of its neighbors in $B_{i}$. Now again applying Lemma 3.7 gives another vertex $v^{\prime}$ with $d_{B_{i}-v-N(y)}\left(v^{\prime}\right)<i$. We can repeat this process until $B_{i} \cap V_{i}$ is empty, at which time each $v \in V_{i}$ has received charge 1. On round $i$, each $v \in V\left(B_{i}\right) \backslash V_{i}$ has lost charge at most $i-1$, since it gave charge 1 to at most $i-1$ neighbors.

Recall that $d(u)+d(v) \geq \Delta(G)+1 \geq \Delta(H)+2$ for each edge $u v \in E(H)$. Thus, on round 2, only $\Delta(H)$-vertices give charge (and only 2-vertices receive it). Analogously, on an arbitrary round $i$, only vertices of degree at least $\Delta(H)+2-i$ give charge. So if a vertex gives charge only on rounds $i$ through 11, then it gives away charge at most $(i-1)+i+\cdots+10$. Since charge is first given on round $\delta(H)$, in general each vertex loses at most $55-(1+2+\cdots+(\delta(H)-2))$. This maximum amount of charge can only be lost by a vertex of degree at least $\Delta(H)-\delta(H)+2$. So, if some vertex of $H$ finishes the discharging rounds with insufficient charge, then $(\Delta(H)-\delta(H)+2)-(55-(1+2+\cdots+(\delta(H)-2))) \leq 11$. This simplifies to $\Delta(H)+\frac{(\delta(H)-2)(\delta(H)-3)}{2} \leq 66$. Thus, if $\delta(H) \geq 5$, we have $\Delta(H)+\delta(H)-2 \leq$ 66 ; if $\delta(H)=4$, then $\Delta(H) \leq 65$. Finally, if $2 \leq \delta(H) \leq 3$, then we still have $\Delta(H) \leq 66$.

Now we are almost done. However, we must still translate our upper bound on $\Delta(H)$ into an upper bound on $\Delta(G)$. Let $u$ be a minimum degree vertex in $H$ and $v$ a neighbor of $u$. Then, in $G$ we have $d_{G}(u v)=d_{H}(u)+d_{H}(v)-1-\mu(u v) \leq \delta(H)+\Delta(H)-2$. Since $G$ is BKfree, every vertex has degree at least $\Delta(G)-1$. So $\Delta(G)-1 \leq \delta(H)+\Delta(H)-2$. Now we apply the bounds from the previous paragraph. If $\delta(H) \geq 5$, then $\Delta(G) \leq \delta(H)+\Delta(H)-1 \leq 67$. If, instead, $\delta(H)=4$, then $\Delta(G) \leq 65+4-1=68$. Finally, if $\delta(H) \leq 3$, then $\Delta(G) \leq$ $66+3-1=68$.

Now we combine Lemmas 3.33 .8 to prove Theorem 3.1. For convenience, we restate it.

Theorem 3.1. If $G$ is a BK-free line graph with $\omega(G)<\Delta(G)$, then $\Delta(G)<69$. Thus, if $G$ is a line graph with $\Delta(G) \geq 69$, then $\chi_{O L}(G) \leq \max \{\omega(G), \Delta(G)-1\}$. 
Proof. Let $G$ be a BK-free graph such that $G$ is the line graph of some graph $H$. First, suppose that $\omega(G)<\Delta(G)$. Now $H$ is 6-degenerate, by Lemma 3.6, so $\operatorname{mad}(H)<12$; thus, the first statement follows from Lemma 3.8. Now consider the second statement. If $\omega(G) \geq \Delta(G)$, then the result holds by Brooks' Theorem (more precisely, its generalization to Alon-Tarsi orientations, proved in [16]). So assume that $G$ is a minimal counterexample; now $\omega(G)<\Delta(G)$ and $\Delta(G) \geq 69$. The minimality of $G$ implies that $G$ is BK-free. Now the first statement implies that $\Delta(G)<69$, which is a contradiction.

Corollary 3.9. If $G$ is a quasi-line graph with $\Delta(G) \geq 69$, then $\chi_{O L}(G) \leq \max \{\omega(G), \Delta(G)-$ $1\}$. Further, if $G$ is a claw-free graph with $\Delta(G) \geq 69$, then $\chi_{\ell}(G) \leq \max \{\omega(G), \Delta(G)-1\}$.

Proof. The first statement follows from Lemma 2.5. The second statement follows from a similar reduction from claw-free graphs to quasi-line graphs for the list-coloring version of the Borodin-Kostochka conjecture, which we proved in [10, Theorem 5.6].

\section{References}

[1] Martin Aigner and Günter M. Ziegler, Proofs from The Book, Third ed., Springer-Verlag, Berlin, 2004, Including illustrations by Karl H. Hofmann.

[2] Noga Alon, Combinatorial nullstellensatz, Combinatorics Probability and Computing 8 (1999), no. 1-2, 7-29.

[3] Noga Alon, Melvyn B. Nathanson, and Imre Ruzsa, The polynomial method and restricted sums of congruence classes, J. Number Theory 56 (1996), no. 2, 404-417.

[4] Noga Alon and Michael Tarsi, Colorings and orientations of graphs, Combinatorica 12 (1992), no. 2, 125-134.

[5] Oleg V. Borodin and Alexandr V. Kostochka, On an upper bound of a graph's chromatic number, depending on the graph's degree and density, Journal of Combinatorial Theory, Series B 23 (1977), no. 2-3, 247-250.

[6] Oleg V. Borodin, Alexandr V. Kostochka, and Douglas R. Woodall, List edge and list total colourings of multigraphs, J. Combin. Theory Ser. B 71 (1997), no. 2, 184-204.

[7] _ On kernel-perfect orientations of line graphs, Discrete Math. 191 (1998), no. 13, 45-49, Graph theory (Elgersburg, 1996).

[8] Maria Chudnovsky and Paul Seymour, The structure of claw-free graphs, Surveys in combinatorics 2005, London Math. Soc. Lecture Note Ser., vol. 327, Cambridge Univ. Press, Cambridge, 2005, pp. 153-171.

[9] _ Claw-free graphs. IV. Decomposition theorem, J. Combin. Theory Ser. B 98 (2008), no. 5, 839-938.

[10] Daniel W. Cranston and Landon Rabern, Coloring claw-free graphs with $\Delta-1$ colors, SIAM J. Discrete Math. 27 (2013), no. 1, 534-549. 
[11] _ Graphs with $\chi=\Delta$ have big cliques, SIAM J. Discrete Math. To appear, preprint available at: http://arxiv.org/abs/1305.3526 (2013).

[12] _ Painting squares in $\Delta^{2}-1$ shades, arXiv preprint, available at: http://arxiv.org/abs/1311.1251 (2013).

[13] Reinhard Diestel, Graph Theory, Fourth ed., Springer Verlag, 2010.

[14] Fred Galvin, The list chromatic index of a bipartite multigraph, J. Combin. Theory Ser. B 63 (1995), no. 1, 153-158.

[15] Dan Hefetz, On two generalizations of the Alon-Tarsi polynomial method, J. Combin. Theory Ser. B 101 (2011), no. 6, 403-414.

[16] Jan Hladkỳ, Daniel Král, and Uwe Schauz, Brooks' Theorem via the Alon-Tarsi Theorem, Discrete Mathematics 310 (2010), no. 23, 3426-3428.

[17] Po-Yi Huang, Tsai-Lien Wong, and Xuding Zhu, Application of polynomial method to on-line list colouring of graphs, European J. Combin. 33 (2012), no. 5, 872-883.

[18] Andrew King, Claw-free graphs and two conjectures on $\omega, \Delta$, and $\chi$, Ph.D. thesis, McGill University, 2009.

[19] Andrew D. King and Bruce A. Reed, Bounding $\chi$ in terms of $\omega$ and $\Delta$ for quasi-line graphs, Journal of Graph Theory 59 (2008), no. 3, 215-228.

[20] Michał Lasoń, A generalization of combinatorial nullstellensatz, Electron. J. of Combin. 17 (2010), no. 1, N32.

[21] Bruce Reed, A strengthening of Brooks' theorem, Journal of Combinatorial Theory, Series B 76 (1999), no. 2, 136-149.

[22] Uwe Schauz, Algebraically solvable problems: describing polynomials as equivalent to explicit solutions, Electron. J. Combin. 15 (2008), no. 1.

[23] - Mr. Paint and Mrs. Correct, Electron. J. Combin. 16 (2009), no. 1, Research Paper 77, 18.

[24] Douglas R. Woodall, The average degree of a multigraph critical with respect to edge or total choosability, Discrete Math. 310 (2010), no. 6-7, 1167-1171. 